\title{
ON THE IRREDUCIBILITY OF ASSOCIATED VARIETIES OF W-ALGEBRAS
}

\author{
TOMOYUKI ARAKAWA AND ANNE MOREAU
}

\begin{abstract}
We investigate the irreducibility of the nilpotent Slodowy slices that appear as the associated variety of $W$-algebras. Furthermore, we provide new examples of vertex algebras whose associated variety has finitely many symplectic leaves.
\end{abstract}

Dedicated to the 60th birthday of Professor Efim Zelmanov

\section{INTRODUCTION}

It is known [Li05] that every vertex algebra $V$ is canonically filtered and therefore it can be considered as a quantization of its associated graded Poisson vertex algebra gr $V$. The Zhu's $C_{2}$-algebra $R_{V}$ of $V$ [Z96] is a generating subalgebra of the differential algebra gr $V$ and has the structure of a Poisson algebra. Its maximal spectrum

$$
X_{V}:=\operatorname{Specm} R_{V}
$$

is called the associated variety of $V$ ([A12]). The associated variety $X_{V}$ is a fundamental invariant of $V$ that captures some important properties of the vertex algebra $V$ itself (see e.g. [BFM, Z96, ABD04, Mi04, A12, A15a, A15b, AM15, AM16].

As a Poisson variety, the associated variety of a vertex algebra is a finite disjoint union of smooth analytic Poisson manifolds, and it is stratified by its symplectic leaves. The case where the associated variety has finitely many symplectic leaves is particularly interesting. This happens for instance when $V$ is an admissible affine vertex algebra ${ }^{1}$ ([A15a]), when $V$ is the simple affine vertex algebra associated with a simple Lie algebra that belongs to the the Deligne exceptional series [D96] at level $k=-h^{\vee} / 6-1$ ([AM15]) with $h^{\vee}$ the dual Coxeter number, or when $V$ is the (generalized) Drinfeld-Sokolov reduction ([FF90, KRW03]) of the latter affine vertex algebras provided that it is nonzero ([A15a]). This is also expected to happen for the vertex algebras obtained from four dimensional $N=2$ superconformal field theories $\left(\left[\mathrm{BLL}^{+} 15\right]\right)$, where the associated variety is expected to coincide with the spectrum of the chiral ring of the Higgs branch of the four dimensional theory. Of course, it also happens when the associated variety of $V$ is a point, that is, when $V$ is lisse (or $C_{2}$-cofinite).

2010 Mathematics Subject Classification. 81R10, 17B08, 14L30.

Key words and phrases. Associated variety of vertex algebras, W-algebras, branching, nilpotent Slodowy slice.

${ }^{1}$ that is, the simple affine vertex algebras associated with admissible representations of affine Kac-Moody algebras. 
In our previous paper [AM16] we conjectured that, under reasonable assumptions on the vertex algebra $V$, the associated variety $X_{V}$ is irreducible if it has only finitely many symplectic leaves (see Conjecture 1).

One of the aims of this paper is to verify this irreducibility conjecture for the known cases where $X_{V}$ has finitely many symplectic leaves. It turns out this is a subtle problem for the associated varieties of (conjecturally simple) $W$-algebras, and some deep results on the geometry of nilpotent orbits are needed for the verifications. More precisely, the associated variety in question has the form

$$
\mathscr{S}_{\mathbb{O}, f}:=\overline{\mathbb{O}} \cap \mathscr{S}_{f},
$$

where $\mathbb{O}$ is a nilpotent orbit in a simple Lie algebra $\mathfrak{g}$ and $\mathscr{S}_{f}$ is the Slodowy slice at a nilpotent element $f$ of $\mathfrak{g}$. The variety $\mathscr{S}_{\mathbb{O}, f}$ is called a nilpotent Slodowy slice ([FJLS15]). It is not irreducible in general. On the other hand, not every nilpotent Slodowy slice appears as the associated variety of some $W$-algebra. We investigate in detail the irreducibility of the variety $\mathscr{S}_{\mathbb{O}, f}$ that appears as the associated variety of a $W$-algebra and confirm the irreducibility for all the known cases.

Another aim of this paper is to provide new examples of vertex algebras whose associated variety has finitely many symplectic leaves. We do this by proving a conjecture stated in our previous article [AM16, Conjecture 2] (Theorem 6.1) and also by showing that the associated variety of the simple affine vertex algebra associated with $\mathfrak{s o}_{2 r+1}$ at level -2 is the short nilpotent orbit closure (Theorem 7.1). Theorems 6.1 and 7.1 give new examples of non-admissible affine vertex algebras whose associated variety is contained in the nilpotent cone.

Acknowledgments. We thank very much Daniel Juteau for his explanations about branchings and nilpotent Slodowy slices. We thank Drazen Adamović for inviting us to the University of Zagreb in June 2016, and the CIRM Luminy for its hospitality during our stay as "Research in pairs" in August, 2016.

The first named author is supported by JSPS KAKENHI Grant Numbers 25287004 and 26610006. The second named author is supported by the ANR Project GeoLie Grant number ANR-15-CE40-0012.

\section{KNOWN EXAMPLES OF VERTEX ALGEBRAs WHOSE ASSOCIATED VARIETy HAS FINITELY MANY SYMPLECTIC LEAVES}

Let $V$ be a vertex algebra, and let

$$
V \rightarrow(\text { End } V)\left[\left[z, z^{-1}\right]\right], \quad a \mapsto a(z)=\sum_{n \in \mathbb{Z}} a_{(n)} z^{-n-1},
$$

be the state-field correspondence. The Zhu's $C_{2}$-algebra is by definition the quotient space $R_{V}=V / C_{2}(V)$, where $C_{2}(V)=\operatorname{span}_{\mathbb{C}}\left\{a_{(-2)} b \mid a, b \in V\right\}$, equipped with the Poisson algebra structure given by

$$
\bar{a} . \bar{b}=\overline{a_{(-1)} b}, \quad\{\bar{a}, \bar{b}\}=\overline{a_{(0)} b},
$$

for $a, b \in V$ with $\bar{a}:=a+C_{2}(V)$. The associated variety $X_{V}$ of $V$ is the reduced scheme of the spectrum of $R_{V}, X_{V}=\operatorname{Specm}\left(R_{V}\right)$.

In $[\mathrm{AM} 16$, Conjecutre $1(2)]$, we stated the following conjecture.

Conjecture 1. Let $V=\oplus_{d \geqslant 0} V_{d}$ be a simple, finitely strongly generated (i.e., $R_{V}$ is finitely generated), positively graded conformal vertex operator algebra such that 
$V_{0}=\mathbb{C}$. Assume that $X_{V}$ has finitely many symplectic leaves. Then $X_{V}$ is irreducible.

In the following we list the known examples of vertex algebras whose associated variety has finitely many symplectic leaves.

2.1. Lisse vertex algebras. Recall [Z96] that a vertex algebra is lisse (or $C_{2}$ cofinite) if $\operatorname{dim} X_{V}=0$. Under the assumptions on $V$ of Conjecture $1, R_{V}=$ $\oplus_{d \geqslant 0}\left(R_{V}\right)_{d}$ and $\left(R_{V}\right)_{0}=\mathbb{C}$, so $X_{V}$ is conic, that is, admits a $\mathbb{C}^{*}$-action that is contracting to a point. Therefore, under the assumptions on $V$ of Conjecture $1, V$ is lisse if and only if $X_{V}=$ \{point $\}$. Hence, if so, $X_{V}$ is a trivial symplectic variety and is irreducible.

2.2. Affine vertex algebras. Let $\mathfrak{g}$ be a complex simple Lie algebra, and $\widehat{\mathfrak{g}}$ the affine Kac-Moody algebra associated with $\mathfrak{g}$ :

$$
\widehat{\mathfrak{g}}=\mathfrak{g} \otimes \mathbb{C}\left[t, t^{-1}\right] \oplus \mathbb{C} K \oplus \mathbb{C} D,
$$

where the commutation relations are given by

$\left[x \otimes t^{m}, y \otimes t^{n}\right]=[x, y] \otimes t^{m+n}+m(x \mid y) \delta_{m+n, 0} K, \quad[K, \widehat{\mathfrak{g}}]=0, \quad\left[D, x \otimes t^{m}\right]=m x \otimes t^{m}$

for $x, y \in \mathfrak{g}, m, n \in \mathbb{Z}$. Here $(\mid)=\frac{1}{2 h^{\vee}} \times$ Killing form of $\mathfrak{g}$. For $k \in \mathbb{C}$, set

$$
V^{k}(\mathfrak{g})=U(\widehat{\mathfrak{g}}) \otimes_{U(\mathfrak{g} \otimes \mathbb{C}[t] \oplus \mathbb{C} K \oplus \mathbb{C} D)} \mathbb{C}_{k},
$$

where $\mathbb{C}_{k}$ is the one-dimensional representation of $\mathfrak{g} \otimes \mathbb{C}[t] \oplus \mathbb{C} K \oplus \mathbb{C} D$ on which $K$ acts as multiplication by $k$ and $\mathfrak{g} \otimes \mathbb{C}[t] \oplus \mathbb{C} D$ acts trivially. As it is well-known $V_{k}(\mathfrak{g})$ is naturally a vertex algebra, called the universal affine vertex algebra associated with $\mathfrak{g}$ at level $k$. The unique simple (graded) quotient $V_{k}(\mathfrak{g})$ is called the simple affine vertex algebra associated with $\mathfrak{g}$ at level $k$.

We have $X_{V^{k}(\mathfrak{g})}=\mathfrak{g}^{*}$, and therefore the associated variety $X_{V_{k}(\mathfrak{g})}$ is a Poisson subscheme of $\mathfrak{g}^{*}$ which is $G$-invariant and conic, where $G$ is the adjoint group of $\mathfrak{g}$. Thus, identifying $\mathfrak{g}$ with $\mathfrak{g}^{*}$ through $(\mid)$, the symplectic leaves of $X_{V_{k}(\mathfrak{g})}$ are exactly the adjoint orbits of $G$ in $X_{V_{k}(\mathfrak{g})}$, and $X_{V_{k}(\mathfrak{g})}$ has finitely many symplectic leaves if and only if $X_{V_{k}(\mathfrak{g})}$ is contained in the nilpotent cone $\mathcal{N}$ of $\mathfrak{g}$.

We list in Table 1 the known pairs $(\mathfrak{g}, k)$ where $X_{V_{k}(\mathfrak{g})} \subset \mathcal{N}$. We do not claim that the list is exhaustive.

\begin{tabular}{|c|c|c|c|}
\hline & type of $\mathfrak{g}$ & $k$ & $X_{V_{k}(\mathfrak{g})}$ \\
\hline$(1)$ & any & $-h^{\vee}$ & $\mathcal{N}$ \\
\hline$(2)$ & any & admissible & $\overline{\mathbb{O}}_{q}$ \\
\hline$(3)$ & $G_{2}$ & -1 & $\overline{\mathbb{O}_{\text {min }}}$ \\
\hline$(4)$ & $D_{4}, E_{6}, E_{7}, E_{8}$ & $k \in \mathbb{Z},-\frac{h^{\vee}}{6}-1 \leqslant k \leqslant-1$ & $\overline{\mathbb{O}_{\text {min }}}$ \\
\hline$(5)$ & $D_{r}$ with $r \geqslant 5$ & $-2,-1$ & $\overline{\mathbb{O}_{\text {min }}}$ \\
\hline$(6)$ & $D_{r}$ with $r$ an even integer & $2-r$ & $\overline{\mathbb{O}_{\left(2^{r-2}, 1^{4}\right)}}$ \\
\hline$(7)$ & $B_{r}$ & -2 & $\overline{\mathbb{O}_{\text {short }}}$ \\
\hline
\end{tabular}

TABle 1. Known pairs $(\mathfrak{g}, k)$ for which $X_{V_{k}(\mathfrak{g})} \subset \mathcal{N}$ 
In case (1) of Table $1, V_{-h^{\vee}}(\mathfrak{g})$ does not satisfy the assumption of Conjecture 1 since it is not conformal, but the irreducibility of $X_{V_{-h} \vee(\mathfrak{g})}$ holds.

In case $(2)$ of Table $1, \mathbb{O}_{q}$ is a nilpotent orbit of $\mathfrak{g}$ described by Tables $2-10$ of [A15a] which only depends on the denominator $q$ of the admissible level $k \in \mathbb{Q}$.

In cases $(3)-(5)$ of Table $1, \mathbb{O}_{\text {min }}$ is the minimal nilpotent orbit of $\mathfrak{g}$.

The statement of the case (6) of Table 1 was conjectured in [AM16] and will be proven in Section 6 . Here $\mathbb{O}_{\left(2^{r-2}, 1^{4}\right)}$ denotes the nilpotent orbit of $\mathfrak{s o}_{2 r}$ corresponding to the partition $\left(2^{r-2}, 1^{4}\right)$ of $2 r$ (see Section 4 ).

In case $(7)$ of Table $1, \mathbb{O}_{\text {short }}$ is the unique short nilpotent orbit in $\mathfrak{s o}_{2 r+1}$. Here by short nilpotent orbit we mean the orbit of a short nilpotent element, and a nilpotent element $e$ of $\mathfrak{g}$ is called short if for $(e, f, h)$ an $\mathfrak{s l}_{2}$-triple,

$$
\mathfrak{g}=\mathfrak{g}_{-1} \oplus \mathfrak{g}_{0} \oplus \mathfrak{g}_{1},
$$

where $\mathfrak{g}_{j}=\{x \in \mathfrak{g} \mid[h, x]=2 j x\}$. The fact that $X_{V_{-2}\left(\mathfrak{s o}_{2 r+1}\right)}=\overline{\mathbb{O}_{\text {short }}}$ is new and will be proven in Theorem 7.1 .

In all the cases of Table $1, X_{V_{k}(\mathfrak{g})}$ is the closure of some nilpotent orbit in $\mathcal{N}$ and so Conjecture 1 holds.

2.3. Simple $W$-algebras. Let $\mathcal{W}^{k}(\mathfrak{g}, f)$ be the affine $W$-algebra associated with a nilpotent element $f$ of $\mathfrak{g}$ defined by the generalized quantized Drinfeld-Sokolov reduction [FF90, KRW03]:

$$
\mathcal{W}^{k}(\mathfrak{g}, f)=H_{D S, f}^{0}\left(V^{k}(\mathfrak{g})\right) .
$$

Here $H_{D S, f}^{\bullet}(M)$ denotes the BRST cohomology of the generalized quantized DrinfeldSokolov reduction associated with $f \in \mathcal{N}$ with coefficients in a $V^{k}(\mathfrak{g})$-module $M$.

We have [DSK06, A15a] a natural isomorphism $R_{\mathcal{W}^{k}(\mathfrak{g}, f)} \cong \mathbb{C}\left[\mathscr{S}_{f}\right]$ of Poisson algebras, so that

$$
X_{\mathcal{W}^{k}(\mathfrak{g}, f)}=\mathscr{S}_{f}
$$

Here $\mathscr{S}_{f}$ is the Slodowy slice at $f$ as in the introduction (cf. Section 3.2). It has a natural Poisson structure induced from that of $\mathfrak{g}^{*}$ ([GG02]).

Let $\mathcal{W}_{k}(\mathfrak{g}, f)$ be the unique simple (graded) quotient of $\mathcal{W}^{k}(\mathfrak{g}, f)$. Then $X_{\mathcal{W}_{k}(\mathfrak{g}, f)}$ is a $\mathbb{C}^{*}$-invariant Poisson subvariety of the Slodowy slice $\mathscr{S}_{f}$.

Let $\mathcal{O}_{k}$ be the category $\mathcal{O}$ of $\widehat{\mathfrak{g}}$ at level $k$. We have a functor

$$
\mathcal{O}_{k} \rightarrow \mathcal{W}^{k}(\mathfrak{g}, f)-\operatorname{Mod}, \quad M \mapsto H_{D S, f}^{0}(M),
$$

where $\mathcal{W}^{k}(\mathfrak{g}, f)$-Mod denotes the category of $\mathcal{W}^{k}(\mathfrak{g}, f)$-modules.

Let $\mathrm{KL}_{k}$ be the full subcategory of $\mathcal{O}_{k}$ consisting of objects $M$ on which $\mathfrak{g}$ acts locally finitely. Note that $V^{k}(\mathfrak{g})$ and $V_{k}(\mathfrak{g})$ are objects of $\mathrm{KL}_{k}$.

Theorem 2.1 ([A15a]). $\quad$ (1) $H_{D S, f}^{i}(M)=0$ for all $i \neq 0, M \in \mathrm{KL}_{k}$. In particular, the functor $\mathrm{KL}_{k} \rightarrow \mathcal{W}^{k}(\mathfrak{g}, f)-\mathrm{Mod}, M \mapsto H_{D S, f}^{0}(M)$, is exact.

(2) For any quotient $V$ of $V^{k}(\mathfrak{g})$,

$$
X_{H_{D S, f}^{0}(V)}=X_{V} \cap \mathscr{S}_{f}
$$

In particular

(a) $H_{D S, f}^{0}(V) \neq 0$ if and only if $\overline{G . f} \subset X_{V}$,

(b) $H_{D S, f}^{0}(V)$ is lisse if $X_{V}=\overline{G . f}$. 
By Theorem $2.1(1), H_{D S, f}^{0}\left(V_{k}(\mathfrak{g})\right)$ is a quotient vertex algebra of $\mathcal{W}^{k}(\mathfrak{g}, f)$ if it is nonzero. Conjecturally [KRW03, KW08], we have

$$
\mathcal{W}_{k}(\mathfrak{g}, f) \cong H_{D S, f}^{0}\left(V_{k}(\mathfrak{g})\right) \text { provided that } H_{D S, f}^{0}\left(V_{k}(\mathfrak{g})\right) \neq 0
$$

(This conjecture has been verified in many cases [A05, A07, A11].)

By Theorem $2.1(2)$, when $X_{V_{k}(\mathfrak{g})} \subset \mathcal{N}$, then $X_{H_{D S, f}^{0}\left(V_{k}(\mathfrak{g})\right)}$ is contained in $\mathscr{S}_{f} \cap \mathcal{N}$ and so has finitely many symplectic leaves.

One of the purposes of the paper is to investigate the irreducibility of $X_{V}$ for $V=H_{D S, f}^{0}\left(V_{k}(\mathfrak{g})\right)$ with $X_{V_{k}(\mathfrak{g})} \subset \mathcal{N}$.

\section{Branching and nilpotent Slodowy slices}

We collect in this section the results that we need about branchings and nilpotent Slodowy slices. We refer to [Gr61, Chap. III, §4.3] for the original definition of unibranchness, and to [KP82] or [FJLS15] for further details on branchings and nilpotent Slodowy slices.

3.1. Smoothly equivalent singularities, cross sections. Consider two varieties $X, Y$ and two points $x \in X, y \in Y$. The singularity of $X$ at $x$ is called smoothly equivalent to the singularity of $Y$ at $y$ if there is a variety $Z$, a point $z \in Z$ and two maps $\varphi: Z \rightarrow X, \psi: Z \rightarrow Y$, such that $\varphi(z)=x, \psi(z)=y$, and $\varphi$ and $\psi$ are smooth in $z$ ([Hes76]). This clearly defines an equivalence relation between pointed varieties $(X, x)$. We denote the equivalence class of $(X, x)$ by $\operatorname{Sing}(X, x)$.

Various geometric properties of $X$ at $x$ only depends on the equivalence class $\operatorname{Sing}(X, x)$, for example: smoothness, normality, seminormality (cf. [KP82, §16.1]), unibranchness (cf. §3.3), Cohen-Macaulay, rational singularities.

Assume that the algebraic group $G$ acts regularly on the variety $X$. Then $\operatorname{Sing}(X, x)=\operatorname{Sing}\left(X, x^{\prime}\right)$ if $x$ and $x^{\prime}$ belongs to the same $G$-orbit $\mathbb{O}$. In this case, we denote the equivalence class also by $\operatorname{Sing}(X, \mathbb{O})$.

A cross section (or transverse slice) at the point $x \in X$ is defined to be a locally closed subvariety $S \subset X$ such that $x \in S$ and the map

$$
G \times S \longrightarrow X, \quad(g, s) \longmapsto g . s,
$$

is smooth at the point $(1, x)$. We have $\operatorname{Sing}(S, x)=\operatorname{Sing}(X, x)$.

In the case where $X$ is the closure of some nilpotent $G$-orbit of $\mathfrak{g}$, there is a natural choice of a cross section as we explain next subsection.

3.2. Nilpotent Slodowy slice. Let $\mathbb{O}, \mathbb{O}^{\prime}$ be two nonzero nilpotent orbits of $\mathfrak{g}$ and pick $f \in \mathbb{O}^{\prime}$. By the Jacobson-Morosov Theorem, we can embed $f$ into an $\mathfrak{s l}_{2}$-triple $(e, h, f)$ of $\mathfrak{g}$. The affine space

$$
\mathscr{S}_{f}:=f+\mathfrak{g}^{e}
$$

is a transverse slice of $\mathfrak{g}$ at $e$, called the Slodowy slice associated with $(e, h, f)$. There is a $\mathbb{C}^{*}$-action on $\mathscr{S}_{f}$ contracting to $f$ (cf. [GG02]). The variety

$$
\mathscr{S}_{\mathbb{O}, f}:=\overline{\mathbb{O}} \cap \mathscr{S}_{f}
$$

is then a transverse slice of $\overline{\mathbb{O}}$ at $f$, which we call, following the terminology of [FJLS15], a nilpotent Slodowy slice.

Note that $\mathscr{S}_{\mathbb{O}, f}=\{f\}$ if and only if $\mathbb{O}=G$.f. Moreover, since the $\mathbb{C}^{*}$-action on $\mathscr{S}_{f}$ is contracting to $f$ and stabilizes $\mathscr{S}_{f, \mathbb{O}}, \mathscr{S}_{\mathbb{O}, f}=\varnothing$ if and only if $G . f \nsubseteq \overline{\mathbb{O}}$. Hence 
we can assume that $\mathbb{O}^{\prime} \subseteq \overline{\mathbb{O}}$, that is, $\mathbb{O}^{\prime} \leqslant \mathbb{O}$ for the Chevalley order on nilpotent orbits. The variety $\mathscr{S}_{\mathbb{O}, f}$ is equidimensional, and

$$
\operatorname{dim} \mathscr{S}_{\mathbb{O}, f}=\operatorname{codim}\left(\mathbb{O}^{\prime}, \overline{\mathbb{O}}\right) .
$$

Since any two $\mathfrak{s l}_{2}$-triples containing $f$ are conjugate by an element of the isotropy group of $f$ in $G$, the isomorphism type of $\mathscr{S}_{\mathbb{O}, f}$ is independent of the choice of such $\mathfrak{s l}_{2}$-triples. Moreover, the isomorphism type of $\mathscr{S}_{\mathbb{O}, f}$ is independent of the choice of $f \in \mathbb{O}^{\prime}$. By focussing on $\mathscr{S}_{\mathbb{O}, f}$, we reduce the study of $\operatorname{Sing}\left(\overline{\mathbb{O}}, \mathbb{O}^{\prime}\right)$ to the study of the singularity of $\mathscr{S}_{\mathbb{O}, f}$ at $f$.

The variety $\mathscr{S}_{\mathbb{O}, f}$ is not always irreducible. We are now interested in sufficient conditions for that $\mathscr{S}_{\mathbb{O}, f}$ is irreducible.

3.3. Branching. Let $X$ be an irreducible algebraic variety, and $x \in X$. We say that $X$ is unibranch at $x$ if the normalization $\pi:(\tilde{X}, x) \rightarrow(X, x)$ of $(X, x)$ is locally a homeomorphism at $x$ [FJLS15, $\S 2.4]$. Otherwise, we say that $X$ has branches at $x$ and the number of branches of $X$ at $x$ is the number of connected components of $\pi^{-1}(x)[\mathrm{BS} 84, \S 5,(\mathrm{E})]$.

As it is explained in [FJLS15, Section 2.4], the number of irreducible components of $\mathscr{S}_{\mathbb{O}, f}$ is equal to the number of branches of $\overline{\mathbb{O}}$ at $f$.

If an irreducible algebraic variety $X$ is normal, then it is obviously unibranch at any point $x \in X$. Hence we obtain the following result.

Lemma 3.1. Let $\mathbb{O}, \mathbb{O}^{\prime}$ be nilpotent orbits of $\mathfrak{g}$, with $\mathbb{O}^{\prime} \leqslant \mathbb{O}$ and $f \in \mathbb{O}^{\prime}$. If $\overline{\mathbb{O}}$ is normal, then $\mathscr{S}_{\mathbb{O}, f}$ is irreducible.

The converse is not true. For instance, there is no branching in type $G_{2}$ but one knowns that the nilpotent orbit $\tilde{A}_{1}$ of $G_{2}$ of dimension 8 is not normal [LS88].

The number of branches of $\overline{\mathbb{O}}$ at $f$, and so the number of irreducible components of $\mathscr{S}_{\mathbb{O}, f}$, can be determined from the tables of Green functions in [Sh80, BS84], as discussed in [BS84, Section 5,(E)-(F)]. We indicate in Table 2 the nilpotent orbits (O) which have branchings in types $F_{4}, E_{6}, E_{7}$ and $E_{8}$ (there is no branching in type $G_{2}$ ). The nilpotent orbits are labelled using the Bala-Carter classification.

\begin{tabular}{|l|l|}
\hline Type $F_{4}$ & $C_{3}, C_{3}\left(a_{1}\right)$. \\
\hline Type $E_{6}$ & $A_{4}, 2 A_{2}, A_{2}+A_{1}$. \\
\hline Type $E_{7}$ & $D_{6}\left(a_{1}\right),\left(A_{5}\right)^{\prime \prime}, A_{4}, A_{3}+A_{2}, D_{4}\left(a_{1}\right)+A_{1}$. \\
\hline Type $E_{8}$ & $E_{7}\left(a_{1}\right), E_{6}, E_{6}\left(a_{1}\right), E_{7}\left(a_{4}\right), A_{6}, D_{6}\left(a_{1}\right), D_{5}+A_{1}, E_{7}\left(a_{5}\right), A_{4}, A_{3}+A_{2}, D_{4}$, \\
& $D_{4}\left(a_{1}\right), A_{3}+A_{1}, 2 A_{2}+A_{1}$. \\
\hline
\end{tabular}

TABLE 2. Branching in exceptional cases

We indicate below the (conjectural) list a non-normal nilpotent orbit closures in the exceptional types. These results are extracted from [LS88, Kr89, B98a, B98b, So03]. The list is known to be exhaustive for the types $G_{2}, F_{4}$ and $E_{6}$. It is only conjecturally exhaustive for the types $E_{7}$ and $E_{8}$.

\section{Normality of nilpotent orbit Closures in Lie algebras of CLASSICAL TYPE}

In view of Lemma 3.1, we recall in this section some useful results about the normality of nilpotent orbit closures in the classical types. The normality question in this setting is now completely answered ([KP79, KP79, So05]). 


\begin{tabular}{|l|l|}
\hline Type $G_{2}$ & $\tilde{A}_{1}$. \\
\hline Type $F_{4}$ & $C_{3}, C_{3}\left(a_{1}\right), \tilde{A}_{2}+A_{1}, \tilde{A}_{2}, B_{2}$. \\
\hline Type $E_{6}$ & $A_{4}, A_{3}+A_{1}, A_{3}, 2 A_{2}, A_{2}+A_{1}$. \\
\hline Type $E_{7}$ & $D_{6}\left(a_{1}\right), D_{6}\left(a_{2}\right),\left(A_{5}\right)^{\prime \prime}, A_{4}, A_{3}+A_{2}, D_{4}\left(a_{1}\right)+A_{1}, A_{3}+2 A_{1}$, \\
& $\left(A_{3}+A_{1}\right)^{\prime},\left(A_{3}+A_{1}\right)^{\prime \prime}, A_{3}$. \\
\hline Type $E_{8}$ & $E_{7}\left(a_{1}\right), E_{7}\left(a_{2}\right), D_{7}\left(a_{1}\right), E_{7}\left(a_{3}\right), E_{6}, D_{6}, E_{6}\left(a_{1}\right), E_{7}\left(a_{4}\right), D_{6}\left(a_{1}\right)$, \\
& $A_{6}, D_{5}+A_{1}, E_{7}\left(a_{5}\right), E_{6}\left(a_{3}\right)+A_{1}, D_{6}\left(a_{2}\right), D_{5}\left(a_{1}\right)+A_{2}, A_{5}+A_{1}$, \\
& $D_{5}, E_{6}\left(a_{3}\right), D_{4}+A_{2}, D_{5}\left(a_{1}\right)+A_{1}, A_{5}, D_{5}\left(a_{1}\right), D_{4}+A_{1}, A_{4}$, \\
& $A_{3}+A_{2}, A_{3}+2 A_{1}, D_{4}, D_{4}\left(a_{1}\right) A_{3}+A_{1}, 2 A_{1}+A_{1}, A_{3}$. \\
\hline
\end{tabular}

TABLE 3. Non-normal nilpotent orbits in exceptional cases

First of all, by [KP79], if $\mathfrak{g}=\mathfrak{s l}_{n}$, then all nilpotent orbit closures are normal. So we only focus on the orthogonal and symplectic Lie algebras. We assume in the rest of the section that $\mathfrak{g}$ is either the Lie algebra $\mathfrak{o}_{n}$ of the orthogonal group $O(n)$, or the Lie algebra $\mathfrak{s o}_{n}$ of the special orthogonal group $S O(n)$, or the Lie algebra $\mathfrak{s p}_{n}$ of the symplectic group $S P(n)$.

4.1. Notations. We fix in this subsection some notations relative to nilpotent orbits in simple Lie algebras of classical type. Our main references are [KP82, CM93]. We follow the notations of [MY16, Appendix]; see therein for more details.

Let $n \in \mathbb{N}^{*}$, and denote by $\mathscr{P}(n)$ the set of partitions of $n$. As a rule, unless otherwise specified, we write an element $\boldsymbol{\lambda}$ of $\mathscr{P}(n)$ as a decreasing sequence $\boldsymbol{\lambda}=$ $\left(\lambda_{1}, \ldots, \lambda_{r}\right)$ omitting the zeroes. Thus,

$$
\lambda_{1} \geqslant \cdots \geqslant \lambda_{r} \geqslant 1 \quad \text { and } \quad \lambda_{1}+\cdots+\lambda_{r}=n .
$$

Let us denote by $\geqslant$ the partial order on $\mathscr{P}(n)$ relative to dominance. More precisely, given $\boldsymbol{\lambda}=\left(\lambda_{1}, \cdots, \lambda_{r}\right), \boldsymbol{\eta}=\left(\mu_{1}, \ldots, \mu_{s}\right) \in \mathscr{P}(n)$, we have $\boldsymbol{\lambda} \geqslant \boldsymbol{\eta}$ if $\sum_{i=1}^{k} \lambda_{i} \geqslant \sum_{i=1}^{k} \mu_{i}$ for $1 \leqslant k \leqslant \min (r, s)$.

Case $\mathfrak{s l}_{n}$. By [CM93, Theorem 5.1.1], nilpotent orbits of $\mathfrak{s l}_{n}$ are parametrized by $\mathscr{P}(n)$. For $\boldsymbol{\lambda} \in \mathscr{P}(n)$, we shall denote by $\mathbb{O}_{\boldsymbol{\lambda}}$ the corresponding nilpotent orbit of $\mathfrak{s l}_{n}$. If $\boldsymbol{\lambda}, \boldsymbol{\eta} \in \mathscr{P}(n)$, then $\mathbb{O}_{\boldsymbol{\eta}} \subset \overline{\mathbb{O}_{\boldsymbol{\lambda}}}$ if and only if $\boldsymbol{\eta} \leqslant \boldsymbol{\lambda}$.

Cases $\mathfrak{o}_{n}$ and $\mathfrak{s o}_{n}$. For $n \in \mathbb{N}^{*}$, set

$$
\mathscr{P}_{1}(n):=\{\boldsymbol{\lambda} \in \mathscr{P}(n) ; \text { number of parts of each even number is even }\} .
$$

By [CM93, Theorems 5.1.2 and 5.1.4], nilpotent orbits of $\mathfrak{s o}_{n}$ are parametrized by $\mathscr{P}_{1}(n)$, with the exception that each very even partition $\boldsymbol{\lambda} \in \mathscr{P}_{1}(n)$ (i.e., $\boldsymbol{\lambda}$ has only even parts) corresponds to two nilpotent orbits. For $\boldsymbol{\lambda} \in \mathscr{P}_{1}(n)$, not very even, we shall denote by $\mathbb{O}_{\mathbf{1}, \boldsymbol{\lambda}}$, or simply by $\mathbb{O}_{\boldsymbol{\lambda}}$ when there is no possible confusion, the corresponding nilpotent orbit of $\mathfrak{s o}_{n}$. For very even $\boldsymbol{\lambda} \in \mathscr{P}_{1}(n)$, we shall denote by $\mathbb{O}_{1, \boldsymbol{\lambda}}^{I}$ and $\mathbb{O}_{1, \boldsymbol{\lambda}}^{I I}$ the two corresponding nilpotent orbits of $\mathfrak{s o}_{n}$. In fact, their union forms a single $O(n)$-orbit. Thus nilpotent orbits of $\mathfrak{o}_{n}$ are parametrized by $\mathscr{P}_{1}(n)$.

If $\boldsymbol{\lambda}, \boldsymbol{\eta} \in \mathscr{P}_{1}(n)$, then $\overline{\mathbb{O}_{1, \boldsymbol{\eta}}^{\bullet}} \subsetneq \overline{\mathbb{O}_{1, \boldsymbol{\lambda}}^{\bullet}}$ if and only if $\boldsymbol{\eta}\left\langle\boldsymbol{\lambda}\right.$, where $\mathbb{O}_{1, \boldsymbol{\lambda}}^{\bullet}$ is either $\mathbb{O}_{1, \boldsymbol{\lambda}}, \mathbb{O}_{1, \boldsymbol{\lambda}}^{I}$ or $\mathbb{O}_{1, \boldsymbol{\lambda}}^{I I}$ according to whether $\boldsymbol{\lambda}$ is very even or not.

Given $\boldsymbol{\lambda} \in \mathscr{P}(n)$, there exists a unique $\boldsymbol{\lambda}^{+} \in \mathscr{P}_{1}(n)$ such that $\boldsymbol{\lambda}^{+} \leqslant \boldsymbol{\lambda}$, and if $\boldsymbol{\eta} \in \mathscr{P}_{1}(n)$ verifies $\boldsymbol{\eta} \leqslant \boldsymbol{\lambda}$, then $\boldsymbol{\eta} \leqslant \boldsymbol{\lambda}^{+}$. 
Case $\mathfrak{s p}_{n}$. For $n \in \mathbb{N}^{*}$, set

$\mathscr{P}_{-1}(n):=\{\boldsymbol{\lambda} \in \mathscr{P}(n)$; number of parts of each odd number is even $\}$.

By [CM93, Theorem 5.1.3], nilpotent orbits of $\mathfrak{s p}_{n}$ are parametrized by $\mathscr{P}_{-1}(n)$. For $\boldsymbol{\lambda}=\left(\lambda_{1}, \ldots, \lambda_{r}\right) \in \mathscr{P}_{-1}(n)$, we shall denote by $\mathbb{O}_{-1, \boldsymbol{\lambda}}$, or simply by $\mathbb{O}_{\boldsymbol{\lambda}}$ when there is no possible confusion, the corresponding nilpotent orbit of $\mathfrak{s p}_{n}$. As in the case of $\mathfrak{s l}_{n}$, if $\boldsymbol{\lambda}, \boldsymbol{\eta} \in \mathscr{P}_{-1}(n)$, then $\mathbb{O}_{-1, \boldsymbol{\eta}} \subset \overline{\mathbb{O}_{-1, \boldsymbol{\lambda}}}$ if and only if $\boldsymbol{\eta} \leqslant \boldsymbol{\lambda}$.

Given $\boldsymbol{\lambda} \in \mathscr{P}(n)$, there exists a unique $\boldsymbol{\lambda}^{-} \in \mathscr{P}_{-1}(n)$ such that $\boldsymbol{\lambda}^{-} \leqslant \boldsymbol{\lambda}$, and if $\boldsymbol{\eta} \in \mathscr{P}_{-1}(n)$ verifies $\boldsymbol{\eta} \leqslant \boldsymbol{\lambda}$, then $\boldsymbol{\eta} \leqslant \boldsymbol{\lambda}^{-}$.

Definition 4.1. Let $\boldsymbol{\lambda} \in \mathscr{P}_{\varepsilon}(n)$. An $\varepsilon$-degeneration of $\boldsymbol{\lambda}$ is an element $\boldsymbol{\eta} \in \mathscr{P}_{\varepsilon}(n)$ such that $\mathbb{O}_{\varepsilon, \boldsymbol{\eta}} \subseteq \overline{\mathbb{O}_{\varepsilon, \boldsymbol{\lambda}}}$, that is, $\boldsymbol{\eta} \leqslant \boldsymbol{\lambda}$.

4.2. Some general facts. Let $\mathbb{O}$ be a nilpotent orbit of $\mathfrak{g}$. Recall that the singular locus of $\overline{\mathbb{O}}$ is $\overline{\mathbb{O}} \backslash \mathbb{O}$. This was shown by Namikawa [N04] using results of Kaledin and Panyushev [Ka06, P91]; see [Hen15, Section 2] for a recent review. This result also follows from Kraft and Procesi's work in the classical types [KP81, KP82], and from the main theorem of [FJLS15] in the exceptional types.

Theorem 4.2 ([KP82, Theorem 1]). Let $\mathbb{O}$ be a nilpotent orbit in $\mathfrak{o}_{n}$ or $\mathfrak{s p}_{n}$.

(1) $\overline{\mathbb{O}}$ is normal if and only if it is unibranch.

(2) $\overline{\mathbb{O}}$ is normal if and only if it is normal in codimension 2.

In particular, $\overline{\mathbb{O}}$ is normal if it does not contain a nilpotent orbit $\mathbb{O}^{\prime} \leqslant \mathbb{O}$ of codimension 2. Theorem 4.2 does not hold if $\mathfrak{g}=\mathfrak{s o}_{2 n}$ and if $\mathbb{O}=\mathbb{O}_{1, \boldsymbol{\lambda}}$, with $\boldsymbol{\lambda}$ very even.

Theorem 4.3 ([Hes79]). Let $\varepsilon \in\{-1,1\}$ and $\boldsymbol{\lambda} \in \mathscr{P}_{\varepsilon}(n)$.

(1) Assume $\mathfrak{g}=\mathfrak{o}_{n}$. If $\lambda_{1}+\lambda_{2} \leqslant 4$, then $\overline{\mathbb{O}_{1, \boldsymbol{\lambda}}}$ is normal.

(2) Assume $\mathfrak{g}=\mathfrak{s p}_{n}$. If $\lambda_{1} \leqslant 2$, then $\overline{\mathbb{O}_{-1, \lambda}}$ is normal.

Theorem 4.3 does not hold if $\mathfrak{g}=\mathfrak{s o}_{2 n}$ and if $\mathbb{O}=\mathbb{O}_{1, \boldsymbol{\lambda}}$ with $\boldsymbol{\lambda}$ very even.

4.3. Minimal $\varepsilon$-degeneration. We present in this subsection the combinatorial method developed in [KP82] to determine the equivalence class $\operatorname{Sing}\left(\overline{\mathbb{O}_{\varepsilon, \boldsymbol{\lambda}}}, \mathbb{O}_{\varepsilon, \boldsymbol{\eta}}\right)$, for $\varepsilon \in\{-1,1\}$ and $\boldsymbol{\eta}<\boldsymbol{\lambda}$.

Definition 4.4 ([KP82, Definition 3.1]). An $\varepsilon$-degeneration $\boldsymbol{\eta} \leqslant \boldsymbol{\lambda}$ is called minimal if $\boldsymbol{\eta} \neq \boldsymbol{\lambda}$ and there is no $\boldsymbol{\nu} \in \mathscr{P}_{\varepsilon}(n)$ such $\boldsymbol{\eta}<\boldsymbol{\nu}<\boldsymbol{\lambda}$ (i.e., $\boldsymbol{\nu}<\boldsymbol{\lambda}$ are adjacent in the ordering on $\left.\mathscr{P}_{\varepsilon}(n)\right)$.

In geometrical terms this means that the orbit $\mathbb{O}_{\varepsilon, \boldsymbol{\eta}}$ is open in the complement of $\mathbb{O}_{\varepsilon, \boldsymbol{\lambda}}$ in $\overline{\mathbb{O}_{\varepsilon, \lambda}}$.

By Theorem 4.2, for the normality question, it is enough to consider minimal $\varepsilon$-degeneration of codimension 2 (except for the very even nilpotent orbits in $\left.\mathfrak{s o}_{2 n}\right)$. Kraft and Procesi introduced a combinatorial equivalence on $\varepsilon$-degenerations [KP82].

Proposition 4.5 ([KP82, Proposition 3.2]). Let $\boldsymbol{\eta} \leqslant \boldsymbol{\lambda}$ be an $\varepsilon$-degeneration. Assume that for two integers $r$ and $s$ the first $r$ rows and the first $s$ columns of $\boldsymbol{\eta}$ and $\boldsymbol{\lambda}$ coincide and that $\left(\lambda_{1}, \ldots, \lambda_{r}\right)$ is an element of $\mathscr{P}_{\varepsilon}\left(\lambda_{1}+\cdots+\lambda_{r}\right)$. Denote by $\boldsymbol{\eta}^{\prime}$ and $\boldsymbol{\lambda}^{\prime}$ be the partitions obtained by erasing these common rows and columns of $\boldsymbol{\eta}$ and $\boldsymbol{\lambda}$ respectively and put $\varepsilon^{\prime}:=(-1)^{s} \varepsilon$. Then $\boldsymbol{\eta}^{\prime} \leqslant \boldsymbol{\lambda}^{\prime}$ is an $\varepsilon^{\prime}$-degeneration and

$$
\operatorname{codim}\left(\mathbb{O}_{\varepsilon^{\prime}, \boldsymbol{\eta}^{\prime}}, \overline{\mathbb{O}_{\varepsilon^{\prime}, \boldsymbol{\lambda}^{\prime}}}\right)=\operatorname{codim}\left(\mathbb{O}_{\varepsilon, \boldsymbol{\eta}}, \overline{\mathbb{O}_{\varepsilon, \boldsymbol{\lambda}}}\right) .
$$


Definition 4.6 ([KP82, Definition 3.3]). In the setting of Proposition 4.5 we say that the $\varepsilon$-degeneration $\boldsymbol{\eta} \leqslant \boldsymbol{\lambda}$ is obtained from the $\varepsilon^{\prime}$-degeneration $\boldsymbol{\eta}^{\prime} \leqslant \boldsymbol{\lambda}^{\prime}$ by adding rows and columns.

An $\varepsilon$-degeneration $\boldsymbol{\eta} \leqslant \boldsymbol{\lambda}$ is called irreducible if it cannot be obtained by adding rows and columns in a non trivial way.

In the setting of Proposition 4.5, when we obtain an irreducible pair $\left(\boldsymbol{\eta}^{\prime}, \boldsymbol{\lambda}^{\prime}\right)$, such a pair is called the type of $\left(\mathbb{O}_{\varepsilon, \boldsymbol{\eta}}, \mathbb{O}_{\varepsilon, \boldsymbol{\lambda}}\right)$.

Remark 4.7. (1) In the previous setting, $\boldsymbol{\eta}^{\prime} \leqslant \boldsymbol{\lambda}^{\prime}$ is minimal if and only if $\boldsymbol{\eta} \leqslant \boldsymbol{\lambda}$ is minimal.

(2) Any $\varepsilon$-degeneration is obtained in a unique way from an irreducible $\varepsilon^{\prime}$ degeneration by adding rows and columns.

So for the classification of the minimal $\varepsilon$-degenerations, one needs to describe the minimal irreducible $\varepsilon$-degenerations. They are given in [KP82, Table 3.4]. We reproduce it (see Table 4) since it will be our main tool in Section 5 . In the sixth line, "codim" refers to the codimension of $\mathbb{O}_{\varepsilon, \boldsymbol{\eta}}$ in $\overline{\mathbb{O}_{\varepsilon, \lambda}}$. The meaning of the last column of the table is explained in [KP82, $\S 14.2$ and 14.3]. For our purpose, what is important is that, except for the type $e$, the singularity of $\overline{\mathbb{O}_{\varepsilon, \lambda}}$ in $\mathbb{O}_{\varepsilon, \boldsymbol{\eta}}$ is normal.

\begin{tabular}{|c|c|c|c|c|c|c|}
\hline Type & Lie algebra & $\varepsilon$ & $\boldsymbol{\lambda}$ & $\boldsymbol{\eta}$ & $\operatorname{codim}$ & $\operatorname{Sing}\left(\overline{\mathbb{O}_{\varepsilon, \boldsymbol{\lambda}}}, \mathbb{O}_{\varepsilon, \boldsymbol{\eta}}\right)$ \\
\hline$a$ & $\mathfrak{s p}_{2}$ & -1 & $(2)$ & $(1,1)$ & 2 & $A_{1}$ \\
$b$ & $\mathfrak{s p}_{2 n}, n>1$ & -1 & $(2 n)$ & $(2 n-1,2)$ & 2 & $D_{n+1}$ \\
$c$ & $\mathfrak{s o}_{2 n+1}, n>0$ & 1 & $(2 n+1)$ & $(2 n-1,1,1)$ & 2 & $A_{2 n-1}$ \\
$d$ & $\mathfrak{s p}_{4 n+2}, n>0$ & -1 & $(2 n+1,2 n+1)$ & $(2 n, 2 n, 2)$ & 2 & $A_{2 n-1}$ \\
$e$ & $\mathfrak{s o}_{4 n}, n>0$ & 1 & $(2 n, 2 n)$ & $(2 n-1,2 n-1,1,1)$ & 2 & $A_{2 n-1} \cup A_{2 n-1}$ \\
$f$ & $\mathfrak{s o}_{2 n+1}, n>1$ & 1 & $\left(2,2,1^{2 n-1}\right)$ & $\left(1^{2 n+1}\right)$ & $4 n-4$ & $b_{n}$ \\
$g$ & $\mathfrak{s p}_{2 n}, n>1$ & -1 & $\left(2,1^{2 n-2}\right)$ & $\left(1^{2 n}\right)$ & $2 n$ & $c_{n}$ \\
$h$ & $\mathfrak{s o}_{2 n}, n>2$ & 1 & $\left(2,2,1^{2 n-4}\right)$ & $\left(1^{2 n}\right)$ & $4 n-6$ & $d_{n}$ \\
\hline
\end{tabular}

TABLE 4. Irreducible minimal $\varepsilon$-degenerations

The main result is the following.

Theorem 4.8 ([KP82, Theorem 12.3]). Let $\boldsymbol{\eta} \leqslant \boldsymbol{\lambda}$ be the $\varepsilon$-degeneration obtained from the $\varepsilon^{\prime}$-degeneration $\boldsymbol{\eta}^{\prime} \leqslant \boldsymbol{\lambda}^{\prime}$ by adding rows and columns. Then

$$
\operatorname{Sing}\left(\overline{\overline{\mathbb{O}}_{\varepsilon, \boldsymbol{\lambda}}}, \mathbb{O}_{\varepsilon, \boldsymbol{\eta}}\right)=\operatorname{Sing}\left(\overline{\overline{\mathbb{O}}_{\varepsilon^{\prime}, \boldsymbol{\lambda}^{\prime}}}, \mathbb{O}_{\varepsilon^{\prime}, \boldsymbol{\eta}^{\prime}}\right) \text {. }
$$

Since very even nilpotent orbits in $\mathfrak{s o}_{2 n}$ do not appear as known associated varieties of vertex algebras, we do not give here the results about the normality question in this case: the question was partially answered in [KP82, Theorem 17.3], the remaining cases were dealt with in [So05].

\section{Irreducibility of nilpotent Slodowy slices as associated Varieties}

Using the results of the previous sections we are now in position to check the following: for any nilpotent orbit $\mathbb{O}$ of $\mathfrak{g}$ appearing in Table 1 , and for any $f \in \overline{\mathbb{O}}$, $\mathscr{S}_{\mathbb{O}, f}$ is irreducible.

We prove the statement by cases in Table 1 .

* Case (1). We have $\mathbb{O}=\mathcal{N}$. Here the result is well-known (or results from Lemma 3.1). 
* Cases (2). First we assume that $\mathfrak{g}$ is of exceptional type. Then we have to check that none of the nilpotent orbits appearing in Tables 4-10 of [A15a] appears in Table 2. We readily verify that it is true and so we are done. Actually, none of these nilpotent orbits (except the nilpotent orbit $\tilde{A}_{1}$ in $G_{2}$ ) even appears in Table 3.

For the classical types, we have more work to do. The result will follow from $§ 5.1$.

* Cases $(3),(4)$ or $(5)$. Here $\mathbb{O}=\mathbb{O}_{\min }$ and so the result is clear since either $f \in \mathbb{O}_{\text {min }}$ or $f=0$.

* Cases $(6)$. Here $\mathbb{O}=\mathbb{O}\left(2^{r-2}, 1^{4}\right)$. According to Theorem $4.3(1), \overline{\mathbb{O}}$ is normal and so it is unibranch by Theorem 4.2 (1). Hence for any $f \in \overline{\mathbb{O}_{\left(2^{r-2}, 1^{4}\right)}}, \mathscr{S}_{\mathbb{O}, f}$ is irreducible.

5.1. Admissible cases in the classical types. In this subsection, we give the necessary data to verify that all nilpotent orbits of Tables $2-3$ of $[\mathrm{A} 15 \mathrm{a}]^{2}$ have normal closures. The case of $\mathfrak{s l}_{n}$ is clear, so we only consider the cases where $\mathfrak{g}=\mathfrak{s p}_{2 r}, \mathfrak{g}=\mathfrak{s o}_{2 r+1}$ and $\mathfrak{g}=\mathfrak{s o}_{2 r}$.

First of all, we observe that no very even nilpotent orbits in $\mathfrak{s o}_{2 r}$ appear in these tables. Then results of Section 4 apply and our strategy is the following.

Let $\boldsymbol{\lambda} \in \mathscr{P}_{\varepsilon}(n)$ be anyone (as in the footnote 2 ) of the partitions appearing in Tables 2-3 of [A15a]. Then consider all minimal $\varepsilon$-degenerations $\boldsymbol{\eta}$ of $\boldsymbol{\lambda}$ such that

$$
\operatorname{codim}\left(\mathbb{O}_{\varepsilon, \boldsymbol{\eta}}, \overline{\mathbb{O}_{\varepsilon, \boldsymbol{\lambda}}}\right)=2 \text {. }
$$

It may happen that there are several such a $\boldsymbol{\eta}$ for a given $\boldsymbol{\lambda}$, or that there is no such a $\boldsymbol{\eta}$ (in this case, $\overline{\mathbb{O}_{\varepsilon, \boldsymbol{\lambda}}}$ does not contain any nilpotent orbit in codimension 2 , and so it is normal).

Then the type of the singularity of $\overline{\mathbb{O}_{\varepsilon, \boldsymbol{\lambda}}}$ in $\mathbb{O}_{\varepsilon, \boldsymbol{\eta}}$ is obtained following the receipt of Proposition 4.5: we erase common rows and common columns in $\boldsymbol{\eta}$ and $\boldsymbol{\lambda}$ in order to get an irreducible pair $\left(\boldsymbol{\lambda}^{\prime}, \boldsymbol{\eta}^{\prime}\right)$ and we set $\varepsilon^{\prime}=(-1)^{s}$, where $s$ is the number of common columns. The type of $\left(\mathbb{O}_{\varepsilon^{\prime}, \boldsymbol{\lambda}^{\prime}}, \mathbb{O}_{\varepsilon^{\prime}, \boldsymbol{\eta}^{\prime}}\right)$ is described in Table 4 and we conclude thanks to Theorem 4.8 .

According to Theorem 4.3, we can assume that $\lambda_{1} \geqslant 3$ ( $\lambda_{1}$ will be $q$ is our tables).

For each $\boldsymbol{\lambda}$ as in Tables 1,2,3 of [A15a] (as in the footnote 2), we present in the Tables 5, 6 and 7 all possible $\boldsymbol{\eta}$ as above, the number $\varepsilon^{\prime}$, the corresponding irreducible pair $\left(\boldsymbol{\lambda}^{\prime}, \boldsymbol{\eta}^{\prime}\right)$, and the type of $\left(\mathbb{O}_{\varepsilon^{\prime}, \boldsymbol{\lambda}^{\prime}}, \mathbb{O}_{\varepsilon^{\prime}, \boldsymbol{\eta}^{\prime}}\right)$ following Table 4.

Case $\mathfrak{g}=\mathfrak{s p}_{2 r}$. Here $\varepsilon=-1$. According to [A15a, Tables 2-3], the different possibilities for $\boldsymbol{\lambda} \in \mathscr{P}_{-1}(2 r)$ are the following:

I: $\boldsymbol{\lambda}=(q, \ldots, q, s), 0 \leqslant s \leqslant q-1, q$ odd, $s$ even.

II: $\boldsymbol{\lambda}=(q, \ldots, q, q-1, s), 0 \leqslant s \leqslant q-1, q$ odd, $s$ even.

III: $\boldsymbol{\lambda}=(q, \ldots, q, s), 0 \leqslant s \leqslant q-1, q$ even, $s$ even.

IV: $\boldsymbol{\lambda}=(q+1, q, \ldots, q, s), 0 \leqslant s \leqslant q-1, q$ odd, $s$ even.

$\mathbf{V}: \boldsymbol{\lambda}=(q+1, q, \ldots, q, q-1, s), 2 \leqslant s \leqslant q-1, q$ odd, $s$ even.

Case $\mathfrak{g}=\mathfrak{s o}_{2 r+1}$. Here $\varepsilon=1$. According to [A15a, Tables 2-3] and the footnote 2, the different possibilities for $\boldsymbol{\lambda} \in \mathscr{P}_{1}(2 r+1)$ are the following:

I: $\boldsymbol{\lambda}=(q, \ldots, q, s), 0 \leqslant s \leqslant q, q$ odd with even multiplicity, $s$ odd.

II: $\boldsymbol{\lambda}=(q, \ldots, q, s, 1), 0 \leqslant s \leqslant q-1, q$ odd with odd multiplicity, $s$ odd.

\footnotetext{
${ }^{2}$ Not every nilpotent orbits of these tables appear as associated variety of admissible vertex algebras. Namely, in Table 2, the case where $q$ is even in $\mathfrak{s o}_{2 r+1}$ does not appear in such a way so we do not consider that case.
} 


\begin{tabular}{|c|c|c|c|c|c|c|}
\hline $\boldsymbol{\lambda}$ & $q, s$ & $\boldsymbol{\eta}$ & $\varepsilon^{\prime}$ & $\boldsymbol{\lambda}^{\prime}$ & $\boldsymbol{\eta}^{\prime}$ & Type \\
\hline $\mathbf{I}$ & $0 \leqslant s \leqslant q-3$ & $(q, \ldots, q, q-1, q-1, s+2)$ & -1 & $(q-s, q-s)$ & $(q-s-1, q-s-1,2)$ & $d$ \\
& $4 \leqslant s \leqslant q-1$ & $(q, \ldots, q, s-2,2)$ & -1 & $(s)$ & $(s-2,2)$ & $b$ \\
& $s=2$ & $(q, \ldots, q, 1,1)$ & -1 & $(2)$ & $(1,1)$ & $a$ \\
$\mathbf{I I}$ & $0 \leqslant s \leqslant q-5$ & $(q, \ldots, q, q-3, s+2)$ & -1 & $(q-1-s)$ & $(q-3-s, 2)$ & $b$ \\
& $s=q-3$ & $(q, \ldots, q, q-2, s+1)$ & -1 & $(2)$ & $(1,1)$ & $a$ \\
& $4 \leqslant s \leqslant q-1$ & $(q, \ldots, q, q-1, s-2,2)$ & -1 & $(s)$ & $(s-2,2)$ & $b$ \\
& $s=2$ & $(q, \ldots, q, q-1,1,1)$ & -1 & $(2)$ & $(1,1)$ & $a$ \\
$\mathbf{I I I}$ & $0 \leqslant s \leqslant q-2$ & $(q, \ldots, q, q-2, s+2)$ & -1 & $(q-s)$ & $(q-s-2,2)$ & $b$ \\
& $s=q-2$ & $(q, \ldots, q, q-1, s+1)$ & -1 & $(2)$ & $(1,1)$ & $a$ \\
& $4 \leqslant s \leqslant q-1$ & $(q, \ldots, q, s-2,2)$ & -1 & $(s)$ & $(s-2,2)$ & $b$ \\
$\mathbf{I V}$ & $s=2$ & $(q, \ldots, q, 1,1)$ & $(2)$ & $(1,1)$ & $a$ \\
& $0 \leqslant s \leqslant q-3$ & $(q+1, q, \ldots, q, q-1, q-1, s+2)$ & -1 & $(q-s, q-s)$ & $(q-s-1, q-s-1,2)$ & $d$ \\
& $4 \leqslant s \leqslant q-1$ & $(q+1, q, \ldots, q, s-2,2)$ & -1 & $(s)$ & $(s-2,2)$ & $b$ \\
$\mathbf{V}$ & $s=2$ & $(q+1, q, \ldots, q, 1,1)$ & -1 & $(2)$ & $(1,1)$ & $a$ \\
& $0 \leqslant s \leqslant q-5$ & $(q+1, q, \ldots, q, q-3, s+2)$ & -1 & $(q-1-s)$ & $(q-3-s, 2)$ & $b$ \\
& $s=q-3$ & $(q+1, q, \ldots, q, q-2, s+1)$ & -1 & $(2)$ & $(1,1)$ & $a$ \\
& $4 \leqslant s \leqslant q-1$ & $(q+1, q, \ldots, q, q-1, s-2,2)$ & -1 & $(s)$ & $(s-2,2)$ & $b$ \\
& $s=2$ & $(q+1, q, \ldots, q, q-1,1,1)$ & -1 & $(2)$ & $(1,1)$ & $a$ \\
\hline
\end{tabular}

TABLE 5. Data for $\mathfrak{g}=\mathfrak{s p}_{2 r}$

III: $\boldsymbol{\lambda}=(q, \ldots, q, s), 0 \leqslant s \leqslant q-1, q$ even, $s$ odd.

IV: $\boldsymbol{\lambda}=(q, \ldots, q, q-1, s, 1), 0 \leqslant s \leqslant q-1, q$ even, $s$ odd.

\begin{tabular}{|c|c|c|c|c|c|c|}
\hline $\boldsymbol{\lambda}$ & $q, s$ & $\boldsymbol{\eta}$ & $\varepsilon^{\prime}$ & $\boldsymbol{\lambda}^{\prime}$ & $\boldsymbol{\eta}^{\prime}$ & Type \\
\hline $\mathbf{I}$ & $0 \leqslant s \leqslant q-4$ & $(q, \ldots, q, q-2, s+2)$ & -1 & $(q-s)$ & $(q-s-2,2)$ & $b$ \\
& $3 \leqslant s \leqslant q$ & $(q, \ldots, q, s-2,1,1)$ & 1 & $(s)$ & $(s-2,1,1)$ & $c$ \\
& $s=q-2$ & $(q, \ldots, q, q-1, q-1)$ & -1 & $(2)$ & $(1,1)$ & $a$ \\
$\mathbf{I I}$ & $0 \leqslant s \leqslant q-4$ & $(q, \ldots, q, q-2, s+2,1)$ & -1 & $(q-s)$ & $(q-s-2,2)$ & $b$ \\
& $5 \leqslant s \leqslant q-1$ & $(q, \ldots, q, s-2,3)$ & -1 & $(s-1)$ & $(s-3,2)$ & $b$ \\
& $s=3$ & $(q, \ldots, q, 2,2)$ & -1 & $(2)$ & $(1,1)$ & $a$ \\
$\mathbf{I I I}$ & $0 \leqslant s \leqslant q-3$ & $(q, \ldots, q, q-1, q-1, s+2)$ & -1 & $(q-s, q-s)$ & $(q-1-s, q-s-1,2)$ & $d$ \\
& $3 \leqslant s \leqslant q$ & $(q, \ldots, q, s-2,1,1)$ & 1 & $(s)$ & $(s-2,1,1)$ & $c$ \\
$\mathbf{I V}$ & $1 \leqslant s \leqslant q-5$ & $(q, \ldots, q, q-3, s+2,1)$ & -1 & $(q-1-s)$ & $(q-3-s, 2)$ & $b$ \\
& $s=q-3$ & $(q, \ldots, q, q-2, s+1,1)$ & -1 & $(2)$ & $(1,1)$ & $a$ \\
& $5 \leqslant s \leqslant q-1$ & $(q, \ldots, q, q-1, s-2,3)$ & -1 & $(s-1)$ & $(s-3,2)$ & $b$ \\
& $s=3$ & $(q, \ldots, q, q-1,2,2)$ & -1 & $(2)$ & $(1,1)$ & $a$ \\
\hline
\end{tabular}

TABLE 6. Data for $\mathfrak{g}=\mathfrak{s o}_{2 r+1}$

Case $\mathfrak{g}=\mathfrak{s o}_{2 r}$. Here $\varepsilon=1$. According to [A15a, Table 2], the different possibilities for $\boldsymbol{\lambda} \in \mathscr{P}_{1}(2 r)$ are the following:

I: $\boldsymbol{\lambda}=(q, \ldots, q, s), 0 \leqslant s \leqslant q, q$ odd with odd multiplicity, $s$ odd.

II: $\boldsymbol{\lambda}=(q, \ldots, q, s, 1), 0 \leqslant s \leqslant q-1, q$ odd with even multiplicity, $s$ odd.

III: $\boldsymbol{\lambda}=(q+1, q, \ldots, q, s), 0 \leqslant s \leqslant q-1, q$ even, $s$ odd.

IV: $\boldsymbol{\lambda}=(q+1, q, \ldots, q, q-1, s, 1), 0 \leqslant s \leqslant q-1, q$ even, $s$ odd.

Remark 5.1. The above verifications show that, except for the non-normal nilpotent orbit $\tilde{A}_{1}$ of dimension 8 in $G_{2}$ that appears as the associated variety of simple affine vertex algebras of type $G_{2}$ at admissible levels with denominator 2 [A15a], all the other nilpotent orbits appearing in Table 1 are actually normal. 


\begin{tabular}{|c|c|c|c|c|c|c|}
\hline $\boldsymbol{\lambda}$ & $q, s$ & $\boldsymbol{\eta}$ & $\varepsilon^{\prime}$ & $\boldsymbol{\lambda}^{\prime}$ & $\boldsymbol{\eta}^{\prime}$ & Type \\
\hline $\mathbf{I}$ & $0 \leqslant s \leqslant q-4$ & $(q, \ldots, q, q-2, s+2)$ & -1 & $(q-s)$ & $(q-s-2,2)$ & $b$ \\
& $3 \leqslant s \leqslant q$ & $(q, \ldots, q, s-2,1,1)$ & 1 & $(s)$ & $(s-2,1,1)$ & $c$ \\
& $s=q-2$ & $(q, \ldots, q, q-1, s+1)$ & -1 & $(2)$ & $(1,1)$ & $a$ \\
$\mathbf{I I}$ & $0 \leqslant s \leqslant q-4$ & $(q, \ldots, q, q-2, s+2,1)$ & -1 & $(q-s)$ & $(q-s-2,2)$ & $b$ \\
& $5 \leqslant s \leqslant q-1$ & $(q, \ldots, q, s-2,3)$ & -1 & $(s-1)$ & $(s-3,2)$ & $b$ \\
& $s=3$ & $(q, \ldots, q, 2,2)$ & -1 & $(2)$ & $(1,1)$ & $a$ \\
$\mathbf{I I I}$ & $3 \leqslant s \leqslant q-1$ & $(q+1, q, \ldots, q, s-2,1,1)$ & 1 & $(s)$ & $(s-2,1,1)$ & $c$ \\
& $0 \leqslant s \leqslant q-3$ & $(q+1, q, \ldots, q, q-1, q-1, s+2)$ & -1 & $(q-s, q-s)$ & $(q-1-s, q-1-s, 2)$ & $d$ \\
& $s=2$ & $(q+1, q, \ldots, q, 1,1)$ & -1 & $(2)$ & $(1,1)$ & $a$ \\
$\mathbf{I V}$ & $0 \leqslant s \leqslant q-5$ & $(q+1, q, \ldots, q, q-3, s+2,1)$ & -1 & $(q-1-s)$ & $(q-3-s, 2)$ & $b$ \\
& $s=q-3$ & $(q+1, q, \ldots, q, q-2, s+1,1)$ & -1 & $(2)$ & $(1,1)$ & $a$ \\
& $5 \leqslant s \leqslant q-1$ & $(q+1, q, \ldots, q, q-1, s-2,3)$ & -1 & $(s-1)$ & $(s-3,2)$ & $b$ \\
& $s=3$ & $(q+1, q, \ldots, q, q-1,2,2)$ & -1 & $(2)$ & $(1,1)$ & $a$ \\
\hline
\end{tabular}

TABLE 7. Data for $\mathfrak{g}=\mathfrak{s o}_{2 r}$

\section{Proof of Conjecture 2 of [AM16]}

In this section we prove the following assertion.

Theorem 6.1 (Conjecture 2 of [AM16]). Let $\mathfrak{g}=\mathfrak{s o}_{2 r}$ with $r$ even, $r \geqslant 4$, and let $k=2-r$. Then

$$
X_{V_{k}(\mathfrak{g})}=\overline{\mathbb{O}_{\left(2^{r-2}, 1^{4}\right)}} .
$$

Proof. Let $\mathfrak{g}=\mathfrak{s o}_{2 r}$ with $r$ even, $r \geqslant 4$. Let $\Delta=\left\{ \pm \varepsilon_{i} \pm \varepsilon_{j} \mid 1 \leqslant i, j \leqslant r, i \neq j\right\}$ be the root system of $\mathfrak{g}$ and take $\Delta_{+}=\left\{\varepsilon_{i} \pm \varepsilon_{j} \mid 1 \leqslant i<j \leqslant r\right\}$ for the set of positive roots.

By Theorem 1.3 of $[\mathrm{AM} 16]$ we know that $X_{V_{k}(\mathfrak{g})} \subset \overline{\mathbb{O}_{\left(2^{r-2}, 1^{4}\right)}}$. Hence it is sufficient to show that $X_{V_{k}(\mathfrak{g})} \supset \overline{\mathbb{O}_{\left(2^{r-2}, 1^{4}\right)}}$. By Theorem 2.1 (1), this is equivalent to that

$$
H_{D S, f}^{0}\left(V_{k}(\mathfrak{g})\right) \neq 0 \quad \text { for } \quad f \in \mathbb{O}_{\left(2^{r-2}, 1^{4}\right)} .
$$

Let $f \in \mathbb{O}_{\left(2^{r-2}, 1^{4}\right)}$, and let $(e, f, h)$ be an $\mathfrak{s l}_{2}$-triple in $\mathfrak{g}$. The weighted Dynkin diagram of $f$ is the following.

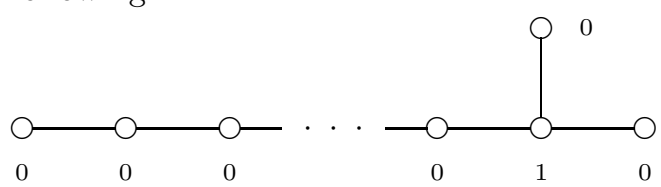

Thus, we may assume that $h=\varpi_{2 r-2}^{\vee}$, where $\varpi_{i}^{\vee}$ is the $i$-th fundamental coweight of $\mathfrak{g}$. We have

$$
\mathfrak{g}=\mathfrak{g}_{-1} \oplus \mathfrak{g}_{-1 / 2} \oplus \mathfrak{g}_{0} \oplus \mathfrak{g}_{1 / 2} \oplus \mathfrak{g}_{1},
$$

where $\mathfrak{g}_{j}=\{x \in \mathfrak{g} \mid[h, x]=2 j x\}$. Set $\Delta_{j}=\left\{\alpha \in \Delta \mid x_{\alpha} \in \mathfrak{g}_{j}\right\}$, so that $\Delta=\bigsqcup_{j} \Delta_{j}$.

Set

$$
D_{h}=D+\frac{1}{2} h,
$$

and put $V_{k}(\mathfrak{g})_{[d]}=\left\{v \in V_{k}(\mathfrak{g}) \mid D_{h} v=d v\right\}$. Since $\alpha\left(D_{h}\right) \geqslant 0$ for any positive real root $\alpha$ of $\widehat{\mathfrak{g}}$ by (1), we have

$$
V_{k}(\mathfrak{g})=\bigoplus_{d \in \frac{1}{2} \mathbb{Z}_{\geqslant 0}} V_{k}(\mathfrak{g})_{[d]} .
$$


The operator $D_{h}$ extends to the grading operator of $\mathcal{W}^{k}(\mathfrak{g}, f)$ as it commutes with the differential of the complex associated with the Drinfeld-Sokolov reduction ([KRW03, A05]). Thus,

$$
H_{D S, f}^{0}\left(V_{k}(\mathfrak{g})\right)=\bigoplus_{d \in \frac{1}{2} \mathbb{Z}_{\geqslant 0}} H_{D S, f}^{0}\left(V_{k}(\mathfrak{g})\right)_{[d]},
$$

where $H_{D S, f}^{0}\left(V_{k}(\mathfrak{g})\right)_{[d]}=\left\{c \in H_{D S, f}^{0}\left(V_{k}(\mathfrak{g})\right) \mid D_{h} c=d c\right\}$.

Now, let $\mathfrak{a}$ be the sualgebra of $\hat{\mathfrak{g}}$ generated by $x_{\alpha} \otimes t, \alpha \in \Delta_{-1}, x_{\beta}, \beta \in \Delta_{0}$, $x_{\gamma} \otimes t^{-1}, \gamma \in \Delta_{1}$. Then $\mathfrak{a}$ is isomorphic to

$$
\mathfrak{g}_{\text {even }}:=\mathfrak{g}_{-1} \oplus \mathfrak{g}_{0} \oplus \mathfrak{g}_{1} \subset \mathfrak{g},
$$

and $\mathfrak{a}$ acts on the each homogeneous subspace $V_{k}(\mathfrak{g})_{[d]}$ as its elements commute with $D_{h}$. We have

$$
\mathfrak{g}_{\text {even }} \cong \mathfrak{s o}_{2(r-2)} \oplus \mathfrak{s l}_{2} \oplus \mathfrak{s l}_{2},
$$

where $\mathfrak{s o}_{2(r-2)}$ is the subalgebra of $\mathfrak{g}$ corresponding to the roots $\pm \epsilon_{i} \pm \epsilon_{j}$ with $1 \leqslant i<j \leqslant r-2$, and $\mathfrak{s l}_{2} \oplus \mathfrak{s l}_{2}$ is the subalgebra corresponding to the roots $\pm \epsilon_{r-1} \pm \epsilon_{r}$.

Let $\mathfrak{p}=\mathfrak{l} \oplus \mathfrak{m}$ be the parabolic subalgebra of $\mathfrak{a}$ whose nilradical $\mathfrak{m}$ is $\left\{x_{\alpha} \otimes t \mid\right.$ $\left.\alpha \in \Delta_{-1}\right\}$. The Levi subalgebra $\mathfrak{l}$ is isomorphic to

$$
\mathfrak{g}_{0} \cong \mathfrak{g l}_{r-2} \oplus \mathfrak{s l}_{2} \oplus \mathfrak{s l}_{2}
$$

The a-module $V_{k}(\mathfrak{g})_{[0]}$ is a highest weight representation of $\mathfrak{a}$ with highest weight $\left((2-r) \varpi_{2(r-2)}, 0,0\right)$ under the isomorphism $\mathfrak{a} \cong \mathfrak{s o}_{2(r-2)} \oplus \mathfrak{s l}_{2} \oplus \mathfrak{s l}_{2}$. This weight verifies the conditions of the Jantzen's simplicity criterion [J77] (see also [Hu08, Section 9.13]). Thus from Jantzen's simplicity criterion one finds that

$$
V_{k}(\mathfrak{g})_{[0]} \cong U(\mathfrak{a}) \otimes_{U(\mathfrak{p})} \mathbb{C}|0\rangle .
$$

The nilpotent element $f \otimes t \in \mathfrak{m} \subset \mathfrak{a}$ belongs to $\mathfrak{s o}_{2(r-2)}$ under the isomorphism $\mathfrak{a} \cong \mathfrak{s o}_{2(r-2)} \oplus \mathfrak{s l}_{2} \oplus \mathfrak{s l}_{2}$, and is a very even nilpotent element that corresponds to the partition $\left(2^{r-2}\right)$. The weighted Dynkin diagram of $f \otimes t$ as nilpotent element of $\mathfrak{s o}_{2(r-2)}$ is given by

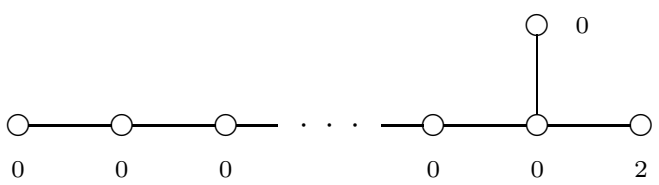

From the definition of the Drinfeld-Sokolov reduction we find that

$$
H_{D S, f}^{0}\left(V_{k}(\mathfrak{g})\right)_{[0]} \cong H_{0}\left(\mathfrak{m}_{-}, V_{k}(\mathfrak{g})_{[0]} \otimes \mathbb{C}_{\chi}\right),
$$

where $\mathfrak{m}_{-}$is the opposite algebra of $\mathfrak{m}$ spanned by $x_{\alpha} \otimes t^{-1}, \alpha \in \Delta_{1}$, so that $\mathfrak{a}=\mathfrak{m}_{-} \oplus \mathfrak{p}$, and $\mathbb{C}_{\chi}$ is the one-dimensional representation of $\mathfrak{m}_{-}$defined by the character $x \mapsto(x \mid f \otimes t)$. Since $V_{k}(\mathfrak{g})_{[0]}$ is a free $U\left(\mathfrak{m}_{-}\right)$-module by (2), it follows that $H_{0}\left(\mathfrak{m}_{-}, V_{k}(\mathfrak{g})_{[0]} \otimes \mathbb{C}_{\chi}\right) \cong H_{0}\left(\mathfrak{m}_{-}, V_{k}(\mathfrak{g})_{[0]}\right) \cong \mathbb{C}$, and therefore $H_{D S, f}^{0}\left(V_{k}(\mathfrak{g})\right)_{[0]}$ is nonzero. Thus, $H_{D S, f}^{0}\left(V_{k}(\mathfrak{g})\right)$ is nonzero, and this completes the proof.

Let $f \in \mathbb{O}_{\left(2^{r-2}, 1^{4}\right)}$. By Theorem 2.1 and Theorem $6.1, \mathcal{W}_{2-r}\left(\mathfrak{s o}_{2 r}, f\right)$, for $r$ even, is lisse. The central charge of $\mathcal{W}_{k}\left(\mathfrak{s o}_{2 r}, f\right)$ is given by

$$
-\frac{(k+r-2)\left(3 k r-6 k+2 r^{2}-12 r+10\right)}{k+2 r-2} .
$$


In particular the central charge of $\mathcal{W}_{2-r}\left(\mathfrak{s o}_{2 r}, f\right)$ is zero. Note that the central charge of $\mathcal{W}_{k}(\mathfrak{g}, f)$ is zero if $\mathcal{W}_{k}(\mathfrak{g}, f)$ is trivial.

Conjecture 2. Let $r$ be even, $r \geqslant 4$, and $f \in \mathbb{O}_{\left(2^{r-2}, 1^{4}\right)}$.

(1) $\mathcal{W}_{2-r}\left(\mathfrak{s o}_{2 r}, f\right)=\mathbb{C}$.

(2) $\mathcal{W}_{k}\left(\mathfrak{s o}_{2 r}, f\right)$ is lisse for any integer $k$ such that $k \geqslant 2-r$.

Parts (1) and (2) of Conjecture 2 are true for $r=4$ by [AM15]. Also, Part (2) of the conjecture is true for $k=2-r$ as mentioned just above.

\section{The ShorT NilPotent ORBit ClOSURE IN TYPE $B$}

In this section, $\mathfrak{g}=\mathfrak{s o}_{2 r+1}$ with $r \geqslant 3$. Let $\Delta=\left\{ \pm \varepsilon_{i} \pm \varepsilon_{j}, \pm \varepsilon_{j} \mid 1 \leqslant i, j \leqslant r, i \neq\right.$ $j\}$ be the root system of $\mathfrak{g}$ and take $\Delta_{+}=\left\{\varepsilon_{i} \pm \varepsilon_{j}, \varepsilon_{k} \mid 1 \leqslant i<j \leqslant r, 1 \leqslant k \leqslant r\right\}$ for the set of positive roots.

Denote by $\left(e_{i}, h_{i}, f_{i}\right)$ the Chevalley generators of $\mathfrak{g}$ in the Bourbaki numbering, and fix the root vectors $e_{\alpha}, f_{\alpha}, \alpha \in \Delta_{+}$so that $\left(h_{i}, i=1, \ldots, r\right) \cup\left(e_{\alpha}, f_{\alpha}, \alpha \in \Delta_{+}\right)$ is a Chevalley basis satisfying the conditions of [Ga82, Chapter IV, Definition 6]. Denote by $\alpha_{1}, \ldots, \alpha_{r}$ the corresponding simple roots of $\mathfrak{g}$. Let $\mathfrak{g}=\mathfrak{n}_{-} \oplus \mathfrak{h} \oplus \mathfrak{n}_{+}$be the corresponding triangular decomposition. For $\alpha \in \Delta_{+}$, denote by $h_{\alpha}=\left[e_{\alpha}, f_{\alpha}\right]$ the corresponding coroot.

Denote by $\varpi_{1}, \ldots, \varpi_{r}$ the fundamental weights and by $\varpi_{1}^{\vee}, \ldots, \varpi_{r}^{\vee}$ the corresponding fundamental co-weights. Note that $\varpi_{i}^{\vee}$ and $\kappa^{\sharp}\left(\varpi_{i}\right)$ are proportional, with $\kappa^{\sharp}: \mathfrak{h}^{*} \rightarrow \mathfrak{h}$ the isomorphism induced from $(\mid)$. In particular $\kappa^{\sharp}\left(\varpi_{1}\right)=\varpi_{1}^{\vee}$.

There is a unique short nilpotent orbit $\mathbb{O}_{\text {short }}$ in $\mathfrak{g}$, which is the nilpotent orbit associated with the $\mathfrak{s l}_{2}$-triple $\left(e_{\theta_{s}}, h_{\theta_{s}}, f_{\theta_{s}}\right)$, where $\theta_{s}$ is the highest short root $\varepsilon_{1}$ and where $h_{\theta_{s}}=2 \varpi_{1}^{\vee}$. So,

$$
\mathfrak{g}=\mathfrak{g}_{-1} \oplus \mathfrak{g}_{0} \oplus \mathfrak{g}_{1},
$$

where $\mathfrak{g}_{j}=\left\{x \in \mathfrak{g} \mid\left[h_{\theta_{s}}, x\right]=2 j x\right\}$. The nilpotent orbit $\mathbb{O}_{\text {short }}$ is labeled by the partition $\left(3,1^{2 r-2}\right)$, and its weighted Dynkin diagram is given by

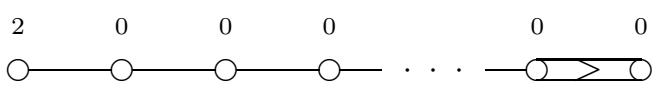

We have

$$
\mathfrak{g}^{\natural}:=\mathfrak{g}_{0}^{f \theta_{s}}=\left\langle e_{\alpha}, f_{\alpha} \mid \alpha=\varepsilon_{i}-\varepsilon_{j}, \varepsilon_{i}+\varepsilon_{j} ; 2 \leqslant i<j \leqslant r\right\rangle \cong \mathfrak{s o}_{2 r-2} .
$$

In particular, $\mathfrak{g}^{\natural}$ is simple. As a module over $\mathfrak{g}^{\natural}=\mathfrak{s o}_{2 r-2}, \mathfrak{g}_{-2}$ decomposes as $\mathfrak{g}_{-2} \cong \mathbb{C} \oplus \mathbb{C}^{2 r-2}$.

In this section we prove the following assertion.

Theorem 7.1. Let $\mathfrak{g}=\mathfrak{s o}_{2 r+1}$, with $r \geqslant 3$, and let $k=-2$. Then

$$
X_{V_{k}(\mathfrak{g})}=\overline{\mathbb{O}_{\text {short }}} \text {. }
$$

Let $w_{2}$ be the singular vector with respect to the adjoint action of $\mathfrak{g}$ as in [AM15, Theorem 4.2] which generates the irreducible representation $W_{2}$ of $\mathfrak{g}$ with highest weight $\theta+\theta_{2}$ in $S^{2}(\mathfrak{g})$, where $S^{2}(\mathfrak{g})$ is the component of $S(\mathfrak{g})$ of degree 2 and $\theta_{2}$ is the highest root of the simple Lie algebra generated by the roots $\alpha_{3}, \ldots, \alpha_{r}$. Namely,

$$
w_{2}=e_{\theta} e_{\theta_{2}}-\sum_{j=1}^{2} e_{\beta_{j}+\theta_{2}} e_{\delta_{j}+\theta_{2}},
$$


with $\beta_{1}:=\alpha_{2}, \delta_{1}:=\alpha_{1}+\alpha_{2}+\alpha_{3}, \beta_{2}:=\alpha_{2}+\alpha_{3}, \delta_{2}:=\alpha_{1}+\alpha_{2}$. Then $\sigma\left(w_{2}\right)$ is a singular vector of $V^{k}(\mathfrak{g})$ if and only if $k=-2$ [AM15, Theorem 4.2] where $\sigma$ is the natural embedding of $\mathfrak{g}$-modules from $S^{2}(\mathfrak{g})$ to $V^{k}(\mathfrak{g})_{2}:=\left\{v \in V^{k}(\mathfrak{g}) \mid D v=-2 v\right\}$ (see [AM15, Lemma 4.1]).

Denote by $V\left(W_{2}\right)$ the zero locus in $\mathfrak{g}^{*} \cong \mathfrak{g}$ of the ideal of $S(\mathfrak{g})$ generated by $W_{2}$.

Lemma 7.2. We have $V\left(W_{2}\right) \cap \mathcal{N} \subset \overline{\mathbb{O}_{\left(3,1^{n-3}\right)}}$.

Proof. We observe that that $\left(3,1^{2 r-2}\right) \in \mathscr{P}_{1}(2 r+1)$ is the unique minimal 1degeneration of $\left(3,2^{2}, 1^{2 r-6}\right) \in \mathscr{P}_{1}(2 r+1)$ (see Definitions 4.1 and 4.4$)$. Therefore, it is enough to show that $V\left(W_{2}\right)$ do not contain $\overline{\mathbb{O}_{\left(3,2^{2}, 1^{2 r-6}\right)}}$. To proceed, we apply [AM16, Lemma 3.3] and we argue as in the proofs of Lemma 7.6 and Lemma 9.2 of [AM16]. Since the verification are similar we omit the details here.

Set $S(\mathfrak{g})^{\mathfrak{h}}=\{x \in S(\mathfrak{g}) \mid[h, x]=0$ for all $h \in \mathfrak{h}\}$ and let $\Psi: S(\mathfrak{g})^{\mathfrak{h}} \rightarrow S(\mathfrak{h})$ be the Chevalley projection map.

Lemma 7.3. The zero weight space of $W_{2}$ has dimension $r(r-1) / 2$.

Proof. It is easy to verify the statement for $r=3$ and $r=4$ where $W_{2}$ has highest weight $2 \varpi_{r}$. For $r \geqslant 5, W_{2}$ has highest weight $\varpi_{4}$. Hence $W_{2} \cong \wedge^{4} \mathbb{C}^{2 r+1}$ and it is easy to show that the zero weight space of $W_{2}$ has dimension $r(r-1) / 2$.

Set $W_{2}^{\mathfrak{h},(r)}:=\Psi\left(W_{2} \cap S(\mathfrak{g})^{\mathfrak{h}}\right)$. By Lemma $7.3, W_{2}^{\mathfrak{h},(r)}$ has dimension $r(r-1) / 2$.

Lemma 7.4. We can describe a set of generators of $W_{2}^{\mathfrak{h},(r)}$ by induction on $r$ as follows. Set

$$
p_{1}^{(3)}:=h_{1} h_{3}, \quad p_{2}^{(3)}:=\left(2 h_{2}+h_{3}\right) h_{3}, \quad p_{3}^{(3)}:=\left(h_{1}+h_{2}\right) h_{2}+\left(h_{2} h_{3}\right) / 2 .
$$

Assume that $r \geqslant 4$ and that generators $p_{1}^{(k)}, \ldots, p_{k(k-1) / 2}^{(k)}$ of $W_{2}^{\mathfrak{h},(k)}$ have been constructed for any $k \in\{3, \ldots, r-1\}$. Set:

$$
\begin{aligned}
& p_{j}^{(r)}:=h_{1} h_{j+2}, j=1, \ldots, r-2, \quad p_{k}^{(r)}:=p_{k}^{(r-1)}, k=r-1, \ldots, r(r-1) / 2-1, \\
& p_{l}^{(r)}:=\left(h_{1}+h_{2}\right) h_{2}+\tilde{p}_{1}^{(r)}, l=r(r-1) / 2,
\end{aligned}
$$

where $\tilde{p}_{1}^{(r)}$ is a homogeneous polynomial of degree 2 in the variables $h_{2}, \ldots, h_{r}$ with no term in $h_{2}^{2}$. Then $p_{1}^{(r)}, \ldots, p_{r(r-1) / 2}^{(r)}$ generate $W_{2}^{\mathfrak{h},(r)}$.

Proof. Since the $r(r-1) / 2$ elements $p_{i}^{(r)}$ as described in the lemma are linearly independent, it suffices to prove that they are elements of $W_{2}^{\mathfrak{h},(r)}$ by Lemma 7.3.

First of all, we have the following relations whose verifications are left to the reader:

$$
\begin{aligned}
& {\left[f_{\beta_{1}+\theta_{2}},\left[f_{\delta_{1}+\theta_{2}}, w_{2}\right]\right] \equiv h_{\delta_{1}+\theta_{2}} h_{\beta_{1}+\theta_{2}}, \quad\left[f_{\delta_{2}+\theta_{2}},\left[f_{\beta_{2}+\theta_{2}}, w_{2}\right]\right] \equiv h_{\delta_{2}+\theta_{2}} h_{\beta_{2}+\theta_{2}},} \\
& {\left[f_{\theta_{2}},\left[f_{\theta}, w_{2}\right]\right] \equiv h_{\theta} h_{\theta_{2}}, \quad\left[f_{\alpha_{4}},\left[f_{\alpha_{3}+\alpha_{4}+2\left(\alpha_{5}+\cdots+\alpha_{r}\right)},\left[f_{\theta}, w_{2}\right]\right]\right] \equiv h_{\theta} h_{\alpha_{4}},} \\
& {\left[f_{\alpha_{3}+\alpha_{4}},\left[f_{\alpha_{4}+2\left(\alpha_{5}+\cdots+\alpha_{r}\right)},\left[f_{\theta}, w_{2}\right]\right]\right] \equiv h_{\theta} h_{\alpha_{3}+\alpha_{4}},}
\end{aligned}
$$

where $\equiv$ means that the equality is modulo $\left(\mathfrak{n}_{-}+\mathfrak{n}_{+}\right) S(\mathfrak{g})$. Moreover, if $r \geqslant 5$ then for any $j=5, \ldots, r-1$ :

$$
\left[f_{\alpha_{4}+\cdots+\alpha_{j}},\left[f_{\alpha_{3}+\cdots+\alpha_{j}+2\left(\alpha_{j+1}+\cdots+\alpha_{r}\right)},\left[f_{\theta}, w_{2}\right]\right]\right] \equiv h_{\theta} h_{\alpha_{4}+\cdots+\alpha_{j}} .
$$


From this, we deduce that the elements

$$
h_{\delta_{1}+\theta_{2}} h_{\beta_{1}+\theta_{2}}, \quad h_{\delta_{2}+\theta_{2}} h_{\beta_{2}+\theta_{2}}, \quad h_{\theta} h_{\theta_{2}}, \quad h_{\theta} h_{\alpha_{4}}, \quad h_{\theta} h_{\alpha_{3}+\alpha_{4}}
$$

are in $W_{2}^{\mathfrak{h},(r)}$. Moreover, if $r \geqslant 5$, then the element $h_{\theta} h_{\alpha_{4}+\cdots+\alpha_{j}}$ is in $W_{2}^{\mathfrak{h},(r)}$ for any $j=5, \ldots, r-1$. For $r \geqslant 5, h_{\theta_{2}}=h_{3}+2 h_{4}+\cdots 2 h_{r-1}+h_{r}$, and for $r=4, h_{\theta_{2}}=$ $h_{3}+h_{4}$. Then, since the elements $h_{\theta} h_{\alpha_{4}+\cdots+\alpha_{r-1}}, h_{\theta} h_{\alpha_{4}+\cdots+\alpha_{j}}-h_{\theta} h_{\alpha_{4}+\cdots+\alpha_{j-1}}$ for $j=5, \ldots, r-1$, and $h_{\theta} h_{\alpha_{3}+\alpha_{4}}-h_{\theta} h_{\alpha_{4}}$ are in $W_{2}^{\mathfrak{h},(r)}$, the elements $h_{\theta} h_{\alpha_{j}}$ also belong to $W_{2}^{\mathfrak{h},(r)}$ for $j=3, \ldots, r$ and $r \geqslant 4$. In conclusion, the elements

$$
h_{\delta_{1}+\theta_{2}} h_{\beta_{1}+\theta_{2}}, \quad h_{\delta_{2}+\theta_{2}} h_{\beta_{2}+\theta_{2}}, \quad h_{\theta} h_{\alpha_{j}}=h_{\theta} h_{j}, \quad j=3, \ldots, r-1,
$$

are in $W_{2}^{\mathfrak{h},(r)}$.

We now prove the statement by induction on $r$.

$* r=3$. Since $q_{1}^{(3)}:=h_{\delta_{1}+\theta_{2}} h_{\beta_{1}+\theta_{2}}, q_{2}^{(3)}:=h_{\delta_{2}+\theta_{2}} h_{\beta_{2}+\theta_{2}}$ and $q_{3}^{(3)}:=h_{\theta} h_{\theta_{2}}$ are in $W_{2}^{\mathfrak{h},(3)}$, the elements $p_{1}^{(3)}=q_{2}^{(3)}-q_{1}^{(3)}=h_{1} h_{3}, p_{2}^{(3)}=q_{3}^{(3)}-h_{1} h_{3}=\left(2 h_{2}+h_{3}\right) h_{3}$ and $p_{3}^{(3)}=\left(q_{1}^{(3)}-q_{3}^{(3)}\right) / 2=\left(h_{1}+h_{2}\right) h_{2}+\left(h_{2} h_{3}\right) / 2$ are in $W_{2}^{\mathfrak{h},(3)}$ too, whence the statement.

$* r=4$. Let $\mathfrak{g}^{\prime}$ be the subalgebra of $\mathfrak{g}$ of type $B_{3}$ generated by $\alpha_{2}, \alpha_{3}, \alpha_{4}$ and assume the statement true for $\mathfrak{g}^{\prime}$. We denote by $w_{2}^{\prime}$ the elements of $S^{2}\left(\mathfrak{g}^{\prime}\right)$ corresponding to $\mathfrak{g}^{\prime}$, and by $\theta^{\prime}, \theta_{2}^{\prime}, \beta_{j}^{\prime}, \delta_{j}^{\prime}, j=1,2$, the roots corresponding to $\theta, \theta_{2}, \beta_{j}, \delta_{j}, j=1,2$, for $\mathfrak{g}^{\prime}$. A direct computation shows that

$$
\left[f_{\alpha_{1}+\alpha_{2}},\left[f_{\alpha_{3}+\alpha_{4}}, w_{2}\right]\right]=e_{\theta^{\prime}} e_{\theta_{2}^{\prime}}-\sum_{k=1}^{2} e_{\beta_{j}^{\prime}+\theta_{2}^{\prime}} e_{\delta_{j}^{\prime}+\theta_{2}^{\prime}}=w_{2}^{\prime} .
$$

It follows from (4) that the elements $p_{1}^{(3)}=h_{2} h_{4}, p_{2}^{(3)}=\left(2 h_{3}+h_{4}\right) h_{4}$ and $p_{3}^{(3)}=$ $\left(h_{2}+h_{3}\right) h_{3}+\left(h_{3} h_{4}\right) / 2$ are in $W_{2}^{\mathfrak{h},(4)}$. Moreover, by (3), since $q_{1}^{(4)}:=h_{\delta_{1}+\theta_{2}} h_{\beta_{1}+\theta_{2}}$, $q_{2}^{(4)}:=h_{\delta_{2}+\theta_{2}} h_{\beta_{2}+\theta_{2}}, q_{3}^{(4)}:=h_{\theta} h_{\alpha_{3}}$ and $q_{4}^{(4)}:=h_{\theta} h_{\alpha_{4}}$ are in $W_{2}^{\mathfrak{h},(4)}$, the elements $q_{2}^{(4)}-q_{1}^{(4)}=h_{1} h_{3}, q_{4}^{(4)}-2 h_{2} h_{4}-\left(2 h_{3}+h_{4}\right) h_{4}=h_{1} h_{4}$ and $q_{1}^{(4)}-h_{1} h_{3}-h_{1} h_{4}=$ $\left(h_{1}+h_{2}\right) h_{2}+\left(h_{3}+h_{4}\right)\left(h_{2}+h_{3}+h_{4}\right)$ are in $W_{2}^{\mathfrak{h},(4)}$ too. Hence the statement is true for $r=4$.

* Assume the statement true for any rank $<r$. Let $\mathfrak{g}^{\prime}$ be the subalgebra of $\mathfrak{g}$ of type $B_{r-1}$ generated by $\alpha_{2}, \ldots, \alpha_{r}$ and assume the statement true for $\mathfrak{g}^{\prime}$. We denote by $w_{2}^{\prime}$ the elements of $S^{2}\left(\mathfrak{g}^{\prime}\right)$ corresponding to $w_{2}$ for $\mathfrak{g}^{\prime}$, and by $\theta^{\prime}, \theta_{2}^{\prime}, \beta_{j}^{\prime}, \delta_{j}^{\prime}, j=1,2$, the roots corresponding to $\theta, \theta_{2}, \beta_{j}, \delta_{j}, j=1,2$, for $\mathfrak{g}^{\prime}$. A direct computation shows that

$$
\left[f_{\alpha_{1}+\alpha_{2}},\left[f_{\alpha_{3}+\alpha_{4}}, w_{2}\right]\right]=e_{\theta^{\prime}} e_{\theta_{2}^{\prime}}-\sum_{k=1}^{2} e_{\beta_{j}^{\prime}+\theta_{2}^{\prime}} e_{\delta_{j}^{\prime}+\theta_{2}^{\prime}}=w_{2}^{\prime} .
$$

From (5), we deduce that the elements $p_{k}^{(r-1)}$, for $k=r-1, \ldots, r(r-1) / 2-1$, viewed as polynomials in the variables $h_{2}, \ldots, h_{r}$, are in $W_{2}^{\mathfrak{h},(r)}$. In addition, by (3), $q_{1}^{(r)}:=h_{\delta_{1}+\theta_{2}} h_{\beta_{1}+\theta_{2}}, q_{2}^{(r)}:=h_{\delta_{2}+\theta_{2}} h_{\beta_{2}+\theta_{2}}, q_{3}^{(r)}:=h_{\theta} h_{\theta_{2}}$ and $q_{j}^{(r)}:=h_{\theta} h_{\alpha_{j}}, j=$ $3, \ldots, r$, are in $W_{2}^{\mathfrak{h},(r)}$. We first deduce that $q_{2}^{(r)}-q_{1}^{(r)}=h_{1} h_{3}$ is in $W_{2}^{\mathfrak{h},(r)}$. Next, for $j=4, \ldots, r$, we have

$$
q_{j}^{(r)}=h_{1} h_{j}+h_{2} h_{j}+\left(h_{2}+2 h_{3}+\cdots+2 h_{r-1}+h_{r}\right) h_{j},
$$


whence $h_{1} h_{j} \in W_{2}^{\mathfrak{h},(r)}$ by the induction hypothesis. Finally, we deduce that $q_{1}^{(r)}-$ $h_{1}\left(h_{3}+2 h_{4}+\cdots+2 h_{r-1}+h_{r}\right)$ is in $W_{2}^{\mathfrak{h},(r)}$ since $h_{1} h_{j} \in W_{2}^{\mathfrak{h},(r)}$ for any $j=3, \ldots, r$. We have proven the expected statement for $r$.

Lemma 7.5. Let $\lambda$ be a nonzero semisimple element of $\mathfrak{g}^{*} \cong \mathfrak{g}$ which belongs to $V\left(W_{2}\right)$. Then either $\lambda \in G$. $\mathbb{C} \varpi_{1}$, or $\lambda \in G$. $\mathbb{C}\left(-\varpi_{i-1}+\varpi_{i}\right)$ for some $i=2, \ldots, r-1$, or $\lambda \in G$. $\mathbb{C}\left(-\varpi_{r-1}+2 \varpi_{r}\right)$. In particular, $\lambda \in V\left(W_{2}\right)$ if and only if $\lambda \in \bigcup_{i=1}^{r} \mathbb{C} \varepsilon_{i}=$ $G \cdot \mathbb{C} \varepsilon_{1}$.

Proof. We have

$$
V\left(W_{2}\right) \cap \mathfrak{h}^{*}=\left\{\lambda \in \mathfrak{h}^{*} \mid p(\lambda)=0 \text { for all } p \in \Psi\left(W_{2} \cap S(\mathfrak{g})^{\mathfrak{h}}\right)\right\} .
$$

Since $V\left(W_{2}\right)$ is $G$-invariant, it is enough to prove the lemma for nonzero elements $\lambda \in V\left(W_{2}\right) \cap \mathfrak{h}^{*}$.

Let $\lambda \in \mathfrak{h}^{*}$ that we write as $\lambda=\sum_{i=1}^{r} \lambda_{i} \varpi_{i}, \lambda_{i} \in \mathbb{C}$. It suffices to prove that if $\lambda \in V\left(W_{2}\right)$, then $\lambda$ is the union of the sets as described in the lemma. Indeed, it is easy to verify that, conversely, these sets all lie in $V\left(W_{2}\right)$.

We prove the statement by induction on $r$. Assume that $\lambda \in V\left(W_{2}\right)$. By Lemma 7.4, we get

$$
\lambda_{1} \lambda_{3}=0, \quad\left(2 \lambda_{2}+\lambda_{3}\right) \lambda_{3}=0, \quad\left(\lambda_{1}+\lambda_{2}\right) \lambda_{2}+\left(\lambda_{2} \lambda_{3}\right) / 2=0 .
$$

So, if $\lambda_{3}=0$, then either $\lambda_{2}=0$, or $\lambda_{1}=-\lambda_{2}$, and if $\lambda_{3} \neq 0$, then $\lambda_{1}=0$ and $\lambda_{3}=-2 \lambda_{2}$, whence the statement.

Assume $r \geqslant 4$ and the statement true for any rank $<r$. We have to solve the systems of equations $p_{i}^{(r)}(z)=0$ for $i=1, \ldots, r(r-1) / 2$. Let $\mathfrak{g}^{\prime}$ be as in the proof of Lemma 7.4. If $\lambda_{1}=0$ then by induction applied to $\mathfrak{g}^{\prime}$, we get the statement. Otherwise, using Lemma 7.4 and the equation $0=p_{j}^{(r)}(\lambda)=\lambda_{1} \lambda_{j+1}$, for $j=1, \ldots, r-2$, we deduce that $\lambda_{3}=\cdots=\lambda_{r}=0$. Therefore, from

$$
0=p_{r(r-1) / 2}^{(r)}(\lambda)=\lambda_{2}\left(\lambda_{1}+\lambda_{2}\right)
$$

we deduce that either $\lambda_{2}=0$ or $\lambda_{1}=-\lambda_{2}$, whence the statement.

The last assertion is straightforward using the description of the fundamental weights $\varpi_{1}, \ldots, \varpi_{r}$.

Denote by $\mathfrak{l}$ the standard Levi subalgebra generated by $\alpha_{2}, \ldots, \alpha_{r}$. Then $\mathfrak{l} \simeq$ $\mathbb{C} \times \mathfrak{s o}_{2 r-2}$ and its center is generated by the semisimple element $\varpi_{1}^{\vee}$. Let

$$
\mathbb{S}_{\text {short }}:=\mathbb{S}_{\mathfrak{l}}
$$

be the Dixmier sheet associated with $\mathfrak{l}$ (cf. [AM16]). It is the the unique sheet containing the nilpotent orbit $\mathbb{O}_{\text {short }}$ of $\mathfrak{s o}_{2 r+1}$. In fact,

$$
\mathbb{O}_{\text {short }}=\operatorname{Ind}_{\mathfrak{l}}^{\mathfrak{g}}(0),
$$

where $\operatorname{Ind}_{\mathfrak{l}}^{\mathfrak{g}}(0)$ is the induced nilpotent orbit of $\mathfrak{g}$ from 0 in $\mathfrak{l}$, and $\mathbb{O}_{\text {short }}$ cannot be induced in another way, see e.g. [MY16, Appendix]. We have (cf. e.g. [AM16])

$$
\overline{\mathbb{S}_{\text {short }}}=\overline{G \cdot\left(\mathbb{C}^{*} \kappa^{\sharp}\left(\varpi_{1}\right)\right)} .
$$

Proposition 7.6. Let $\mathfrak{g}, k$ be as in Theorem \%.1. Then $X_{V_{k}(\mathfrak{g})} \subset \overline{\mathbb{S}_{\text {short }}}$. 
Proof. Let $\tilde{V}_{k}(\mathfrak{g})$ be the quotient of $V^{k}(\mathfrak{g})$ by the submodule generated by $\sigma\left(w_{2}\right)$. Then $X_{\tilde{V}_{k}(\mathfrak{g})}$ is the zero locus of the ad $\mathfrak{g}$-submodule of $S(\mathfrak{g})$ generated by the image of $w_{2}$ in $R_{V_{k}(\mathfrak{g})}=S(\mathfrak{g})$, that is, $V\left(W_{2}\right)$. By Lemma 7.2, Lemma 7.5 and [AM16, Lemma $2.1(2)]$, we obtain that $X_{\tilde{V}_{k}(\mathfrak{g})}=\overline{\mathbb{S}_{\text {short }}}$. This completes the proof as $V_{k}(\mathfrak{g})$ is a quotient of $\tilde{V}_{k}(\mathfrak{g})$.

Proposition 7.7. Let $\mathfrak{g}, k$ be as in Theorem 7.1. Then $H_{f_{\theta_{s}}^{0}}^{0}\left(V_{k}(\mathfrak{g})\right)$ is nonzero and simple. In particular,

$$
X_{V_{k}(\mathfrak{g})} \supset \overline{\mathbb{O}_{\text {short }}} \text {. }
$$

Proof. Consider the subalgebra $\mathfrak{a}$ of $\widehat{\mathfrak{g}}$ generated by $x_{\alpha} \otimes t, \alpha \in \Delta_{-1}, x_{\beta}, \beta \in \Delta_{0}$, $x_{\gamma} \otimes t^{-1}, \gamma \in \Delta_{1}$, which is isomorphic to $\mathfrak{g}$. The vacuums vector $|0\rangle$ generates the irreducible representation of $\mathfrak{a}=\mathfrak{g}$ with highest weight $-2 \varpi_{1}$. Then as in the proof of Theorem 6.1, we use Jantzen's simplicity criterion [J77] (see also [Hu08, Section 9.13]) to find that $U(\mathfrak{a})|0\rangle \cong U(\mathfrak{a}) \otimes_{U(\mathfrak{p})} \mathbb{C}|0\rangle$, where $\mathfrak{p}$ is the parabolic subalgebra of $\mathfrak{a}$ generated by $x_{\alpha} \otimes t, \alpha \in \Delta_{-1}, x_{\beta}, \beta \in \Delta_{0}$. It follows from [AM16, Theorem 6.3] that $H_{f_{\theta_{s}}}^{0}\left(V_{k}(\mathfrak{g})\right)$ is nonzero and almost irreducible. As $H_{f_{\theta_{s}}}^{0}\left(V_{k}(\mathfrak{g})\right)$ is cyclic, the almost irreduciblity is the same as the simplicity. Finally, the last assertion follows from Theorem 2.1 (2).

Lemma 7.8. Let $Y$ be a $G$-invariant subvariety of $\overline{\mathbb{S}_{\text {short }}}$ containing $\overline{\mathbb{O}_{\text {short }}}$. Then $Y=\overline{\mathbb{S}_{\text {short }}}$ or $Y=\overline{\mathbb{O}_{\text {short }}}$.

Proof. By [AM16, Lemma 2.1], $\overline{\mathbb{S}_{\text {short }}}=G \cdot \mathbb{C}^{*} \lambda \cup \overline{\overline{\mathbb{O}}_{\text {short }}}$.

Let $\operatorname{Vir}^{c}$ denote the universal Virasoro vertex algebra at central charge $c \in \mathbb{C}$, and $\operatorname{Vir}_{c}$ the unique simple quotient of $\operatorname{Vir}^{c}$. We know that the following conditions are equivalent ([BFM, W93], see also [A12, A15b]):

(1) $\operatorname{Vir}_{c}$ is rational,

(2) $\operatorname{Vir}_{c}$ is lisse,

(3) $c=c_{p, q}:=1-6(p-q)^{2} / p q$ with $p, q \in \mathbb{Z}_{\geqslant 2},(p, q)=1$.

Theorem 7.9. Let $\mathfrak{g}, k$ be as in Theorem 7.1. We have $H_{f_{\theta_{s}}}^{0}\left(V_{k}(\mathfrak{g})\right) \cong \operatorname{Vir}_{c_{2,2 r-3}}$. Thus, $\mathcal{W}_{k}\left(\mathfrak{g}, f_{\theta_{s}}\right) \cong \operatorname{Vir}_{c_{2,2 r-3}}$. In particular $\mathcal{W}_{k}\left(\mathfrak{g}, f_{\theta_{s}}\right)$ is lisse and rational.

Proof. First it is straightforward to check that $H_{f_{\theta_{s}}}^{0}\left(V_{k}(\mathfrak{g})\right)$ and $\operatorname{Vir}_{c_{2,2 r-3}}$ has the same central charge.

Let $\tilde{V}_{k}(\mathfrak{g})$ be as in the proof of Proposition 7.6. Then $X_{H_{D S, f_{\theta_{s}}}^{0}\left(\tilde{V}_{k}(\mathfrak{g})\right)}=\overline{\mathbb{S}_{\text {short }}} \cap$ $\mathscr{S}_{f_{\theta_{s}}}$ by Theorem 2.1. Since $f_{\theta_{s}}+e_{\theta_{s}}$ is a semisimple element that is conjugate to $h_{\theta_{s}}$, we have (see [AM16, Remark 3.5]),

$$
X_{H_{D S, f_{\theta_{s}}}^{0}\left(\tilde{V}_{k}(\mathfrak{g})\right)}=f_{\theta_{s}}+\mathbb{C} e_{\theta_{s}} .
$$

This implies that $R_{H_{D S, f_{\theta_{s}}}^{0}}\left(\tilde{V}_{k}(\mathfrak{g})\right)$ is the polynomial ring generated by the image of the conformal vector $L$ of $H_{D S, f_{\theta_{s}}}^{0}\left(\tilde{V}_{k}(\mathfrak{g})\right)$. Hence, $H_{D S, f_{\theta_{s}}}^{0}\left(\tilde{V}_{k}(\mathfrak{g})\right)$ is strongly generated by $L$. As $V_{k}(\mathfrak{g})$ is a quotient of $\tilde{V}_{k}(\mathfrak{g})$, the exactness of $H_{D S, f_{\theta_{s}}}^{0}($ ) (Theorem $2.1(1))$ implies that $H_{D S, f_{\theta_{s}}}^{0}\left(V_{k}(\mathfrak{g})\right)$ is also strongly generated by its conformal vector. Since it is simple by Proposition 7.7, $H_{D S, f_{\theta_{s}}}^{0}\left(V_{k}(\mathfrak{g})\right)$ must be isomorphic to $\operatorname{Vir}_{c_{2,2 r-3}}$. 
Proof of Theorem 7.1. By Proposition 7.6 and Proposition 7.7, $X_{V_{k}(\mathfrak{g})}$ is a $G$ invariant subvariety of $\overline{\mathbb{S}_{\text {short }}}$ containing $\overline{\mathbb{O}_{\text {short }}}$. Thus $X_{V_{k}(\mathfrak{g})}=\overline{\mathbb{S}_{\text {short }}}$ or $X_{V_{k}(\mathfrak{g})}=$ $\overline{\mathbb{O}_{\text {short }}}$ by Lemma 7.8. But if $X_{V_{k}(\mathfrak{g})}=\overline{\mathbb{S}_{\text {short }}}$ then $X_{H_{D S, f_{\theta_{s}}}\left(\tilde{V}_{k}(\mathfrak{g})\right)}=\overline{\mathbb{S}_{\text {short }}} \cap \mathscr{S}_{f_{\theta_{s}}}$ is one-dimensional and $H_{D S, f_{\theta_{s}}}\left(V_{k}(\mathfrak{g})\right)$ cannot be lisse, which contradicts Theorem 7.9, whence $X_{V_{k}(\mathfrak{g})}=\overline{\mathbb{O}_{\text {short }}}$.

Conjecture 3. Let $\mathfrak{g}=\mathfrak{s o}_{2 r+1}, r \geqslant 3$. Then $\mathcal{W}_{k}\left(\mathfrak{g}, f_{\theta_{s}}\right)$ is lisse for any integer $k$ such that $k \geqslant-2$.

Conjecture 3 is true for $k=-2$ by Theorem 7.1.

\section{REFERENCES}

[ABD04] T. Abe, G. Buhl, and C. Dong. Rationality, regularity, and $C_{2}$-cofiniteness. Trans. Amer. Math. Soc., 356(8):3391-3402 (electronic), 2004.

[A05] T. Arakawa. Representation theory of superconformal algebras and the Kac-RoanWakimoto conjecture. Duke Math. J., 130(3):435-478, 2005.

[A07] T. Arakawa. Representation theory of $W$-algebras. Invent. Math., 169(2):219-320, 2007

[A11] T. Arakawa. Representation theory of $W$-algebras, II. volume 61 of Adv. Stud. Pure Math., pages 51-90. Math. Soc. Japan, Tokyo, 2011.

[A12] T. Arakawa. A remark on the $C_{2}$ cofiniteness condition on vertex algebras. Math. Z., 270(1-2):559-575, 2012.

[A15a] T. Arakawa. Associated varieties of modules over Kac-Moody algebras and $C_{2}$ cofiniteness of $W$-algebras. Int. Math. Res. Not. 2015(22): 11605-11666, 2015.

[A15b] T. Arakawa. Rationality of W-algebras: principal nilpotent cases. Ann. Math., 182(2):565-694, 2015.

$[\mathrm{BFM}]$ A. Beilinson, B. Feigin, and B. Mazur. Introduction to algebraic field theory on curves. preprint.

[AM15] T. Arakawa and A. Moreau. Joseph ideals and lisse minimal $W$-algebras. J. Inst. Math. Jussieu, published online, arXiv:1506.00710[math.RT].

[AM16] T. Arakawa and A. Moreau. Sheets and associated varieties of affine vertex algebras. arXiv:1601.05906[math.RT].

$\left[\mathrm{BLL}^{+} 15\right]$ C. Beem, M. Lemos, P. Liendo, W. Peelaers, L. Rastelli, and B. van Rees. Infinite chiral symmetry in four dimensions. Comm. Math. Phys., 336(3):1359-1433, 2015.

[BS84] W. Beynon and N. Spaltenstein. Green functions of finite Chevalley groups of type $\mathrm{E}_{n}$ $(n=6,7,8)$. J. Algebra 88(2):584-614, 1984.

[B98a] A. Broer. Decomposition varieties in semisimple Lie algebras. Canad. J. Math., 50(5):929-971, 1998.

[B98b] A. Broer. Normal nilpotent varieties in $F_{4}$. J. Algebra, 207(2):427-448, 1998.

[CM93] D. Collingwood and W.M. McGovern. Nilpotent orbits in semisimple Lie algebras. Van Nostrand Reinhold Co. New York, 65, 1993.

[D96] P. Deligne. La série exceptionnelle de groupes de Lie. C. R. Acad. Sci. Paris, Ser I, 322(4), 321-326, 1996.

[DSK06] A. De Sole and V. Kac. Finite vs affine $W$-algebras. Japan. J. Math., 1(1):137-261, 2006.

[FF90] B. Feigin and E. Frenkel. Quantization of the Drinfel'd-Sokolov reduction. Phys. Lett. $B, 246(1-2): 75-81,1990$.

[FJLS15] B. Fu, D. Juteau, P. Levy and E. Sommers. Generic singularities of nilpotent orbit closures. arXiv:1502.05770[math.RT], to appear in Adv. Math..

[GG02] W.L. Gan and V. Ginzburg. Quantization of Slodowy slices. Int. Math. Res. Not., (5):243-255, 2002.

[Ga82] D. Garfinkle. A new construction of the Joseph ideal. PhD thesis, MIT, 1982

[Gr61] A. Grothendieck. Éléments de géométrie algébrique. III. Étude cohomologique des faisceaux cohérents. I. Inst. Hautes Études Sci. Publ. Math. 11(1):5-167, 1961.

[Hen15] A. Henderson. Singularities of nilpotent orbit closures. Rev. Roumaine Math. Pures Appl. 60(4):441-469, 2015. 
[Hes76] W. Hesselink. Singularities in the nilpotent scheme of a classical group. Trans. Amer. Math. Soc. 222:1-32, 1976.

[Hes79] W. Hesselink. The normality of closures of orbits in a Lie algebra. Comment. Math. Helv., 54(1):105-110, 1979.

[Hu08] J. Humphreys. Representations of semisimple Lie algebras in the BGG category $\mathcal{O}$. Graduate Studies in Mathematics, 94. American Mathematical Society, Providence, RI, 2008

[J77] J.C. Jantzen. Kontravariante Formen auf induzierten Darstellungen halbeinfacher LieAlgebren. Math. Ann., 226(1):53-65, 1977.

[KRW03] V. Kac, S. Roan, and M. Wakimoto. Quantum reduction for affine superalgebras. Comm. Math. Phys., 241(2-3):307-342, 2003.

[KW08] V. Kac and M. Wakimoto. On rationality of $W$-algebras. Transform. Groups, 13(34):671-713, 2008.

[Ka06] D. Kaledin. Symplectic singularities from the Poisson point of view. J. Reine Angew. Math., 600:135-156, 2006.

[Kr89] H. Kraft. Closures of conjugacy classes in $G_{2}$. J. Algebra, 126(2):454-465, 1989.

[KP79] H. Kraft and C. Procesi. Closures of conjugacy classes of matrices are normal. Invent. Math. 53(3):227-247, 1979.

[KP81] H. Kraft and C. Procesi. Minimal singularities in $G L_{n}$. Invent. Math. 62(3):503-515, 1981.

[KP82] H. Kraft and C. Procesi. On the geometry of conjugacy classes in classical groups. Comment. Math. Helv. 57(4):539-602, 1982.

[LS88] T. Levasseur and S.P. Smith. Primitive ideals and nilpotent orbits in type $G_{2}$. J. Algebra 114(1):81-105, 1988.

[Li05] H. Li. Abelianizing vertex algebras. Comm. Math. Phys., 259(2):391-411, 2005.

[Mi04] M. Miyamoto. Modular invariance of vertex operator algebras satisfying $C_{2}$ cofiniteness. Duke Math. J., 122(1):51-91, 2004.

[MY16] A. Moreau and R.W.T. Yu. Jet schemes of the closure of nilpotent orbits. Pacific J. Math., 281(1):137-183, 2016.

[N04] Y. Namikawa, Birational geometry of symplectic resolutions of nilpotent orbits, Moduli spaces and arithmetic geometry, 75-116. Adv. Stud. Pure Math., 45, Math. Soc. Japan, Tokyo, 2006 preprint http://arxiv.org/pdf/math/0408274v1.pdf.

[P91] D. Panyushev. Rationality of singularities and the Gorenstein property for nilpotent orbits. Functional Analysis and Its Applications, 25(3):225-226, 1991.

[Sh80] T. Shoji. On the Springer representations of Chevalley groups of type $F_{4}$. Comm. Algebra 8(5):409-440, 1980.

[So03] E. Sommers. Normality of nilpotent varieties in $E_{6}$. J. Algebra 270(1):288-306, 2003.

[So05] E. Sommers. Normality of very even nilpotent varieties in $D_{2 l}$. Bull. London Math. Soc. 37(3):351-360, 2005.

[W93] W. Wang. Rationality of Virasoro vertex operator algebras. Internat. Math. Res. Notices, (7):197-211, 1993.

[Z96] Y. Zhu. Modular invariance of characters of vertex operator algebras. J. Amer. Math. Soc., 9(1):237-302, 1996.

Department of Mathematics, Mit, 77 Massachusetts Ave. Cambridge MA 02139 USA

Research Institute for Mathematical Sciences, Kyoto University, Kyoto 606-8502 JAPAN

E-mail address: arakawa@kurims.kyoto-u.ac.jp

Laboratoire de Mathématiques et Applications, Téléport 2 - BP 30179, Boulevard Marie et Pierre Curie, 86962 Futuroscope Chasseneuil Cedex, France

E-mail address: anne.moreau@math.univ-poitiers.fr 